UCRL-20001

\title{
COMPILATION OF ELASTIC SCATTERING DATA
}

\author{
Geoffrey C. Fox and C. Quigg \\ January 1970 \\ Lawrence Radiation Laboratory \\ University of California \\ Berkeley, California 94720
}

Printed in the U.S.A. at Lawrence Radiation Laboratory, Berkeley, and in Europe at CERN, Geneva. 


\section{DISCLAIMER}

This report was prepared as an account of work sponsored by an agency of the United States Government. Neither the United States Government nor any agency Thereof, nor any of their employees, makes any warranty, express or implied, or assumes any legal liability or responsibility for the accuracy, completeness, or usefulness of any information, apparatus, product, or process disclosed, or represents that its use would not infringe privately owned rights. Reference herein to any specific commercial product, process, or service by trade name, trademark, manufacturer, or otherwise does not necessarily constitute or imply its endorsement, recommendation, or favoring by the United States Government or any agency thereof. The views and opinions of authors expressed herein do not necessarily state or reflect those of the United States Government or any agency thereof. 


\section{DISCLAIMER}

Portions of this document may be illegible in electronic image products. Images are produced from the best available original document. 


\section{PAGES $\mathrm{i}$ to $\mathrm{ii}$ WERE INTENTIONALLY LEFT BLANK}


Geoffrey C. Fox

Cavendish Laboratory, University of Cambridge

Cambridge, England

and

C. Quigg

Lawrence Radiation Laboratory, University of California Berkeley, California 94720

\begin{abstract}
We present data for clastic scattering of $\pi^{+}$, $\pi^{-}, K^{+}, K^{-}, p$, and $\bar{p}$ on protons in figures and tables.
\end{abstract}

This report was prepared as an account of work sponsored by the United States Government. Neither the United States nor the United States Atomic Energy Commission, nor any of their employees, nor any of their contractors, subculladius, ir thcir omployeos, makes any warranty, express or implied, or assumes any legal liability or responsibility for the accuracy, completeness or usefulness of any information, apparatus, product or process disclosed; or represents that its use would not infringe privately owned rights.

This work was supported in part by the U.S. Atomic Energy Commission. 
This document contains high energy elastic scattering data which have been of interest to us in the. study of elementary particle collisions. It is produced with the blessing, but without the guarantee, of the Particle Data Group, who view it as an interim measure, to serve until their more systematic collections appear. We can promise neither completeness nor perfect accuracy. On the other hand we have accumulated these data for our own use in the past few years, with the care one reserves for personal treasures.

Our figures give differential cross sections, total cross sections, and "effective alphas", or effective Regge trajectories: $\alpha_{\text {eff }}$. These last are derived from the data by (interpolating in $t$, if necessary and) fitting do/dt from all energies s, at each particular value of $t$, to the form

$$
\frac{d \sigma}{d t}(s ; t \text { fixed })=f(t)\left(\frac{s}{s_{0}}\right)^{2 \alpha} \text { eff }(t)-2
$$

with $s_{j}=1(\mathrm{GeV} / \mathrm{c})^{2}$.

The tables give differential cross sections and total cross sections in $\mathrm{mb} /(\mathrm{GeV})^{2}$ and $\mathrm{mb}$, respectively; polarization, and (real part of amplitude)/(imaginary part of amplitude).

\section{ACKNOWLEDGMENT}

We thank Professor Arthur Rosenfeld for suggesting this paper and Dr. LeRoy Price for technical assistance and advice. Dr. Donald Austin and Dr. William Greiman provided the figure of $\alpha_{\text {eff }}$ for $\overline{p p}$ scattering. We are grateful to the Thompson Chemistry Group at IRL for the use of their PDP-9 computer for editing, and to Elizabeth Quigg for help with that task: 
Fig. 1. do/dt, $\pi^{+} p$ elastic. Successive sets of data are spaced by one decade, and are identified by $p_{l a b}$ in $\mathrm{GeV} / \mathrm{c}$.

Fig. 2. do/dt, $\pi^{-p}$ elastic.

Fig. 3. do/dt, $\mathrm{K}^{-\mathrm{p}}$ elastic.

Fig. 4. do/dt, $K^{+} \mathrm{p}$ elastic.

Fig. 5. do/dt, pp elastic.

Fig. 6. do/dt, $\bar{p} p$ elastic.

Fig. 7. Total $\pi^{ \pm} p$ cross sections.

Fig. 8. Total $K^{ \pm} \mathrm{p}$ cross sections.

Fig. 9. Total $\mathrm{pp}, \overline{\mathrm{p}}$. cross sections.

Fig. 10. $\alpha_{\text {eff }}, \pi^{ \pm}$p elastic.

Fig. 1l. $\alpha_{\text {eff }}, K^{ \pm} p$ elastic.

Fig. 12. $\alpha_{\text {eff' }}$ pp elastic.

Fig. 13. $\alpha_{\text {cff }}, \overline{p p}$ elastic. 

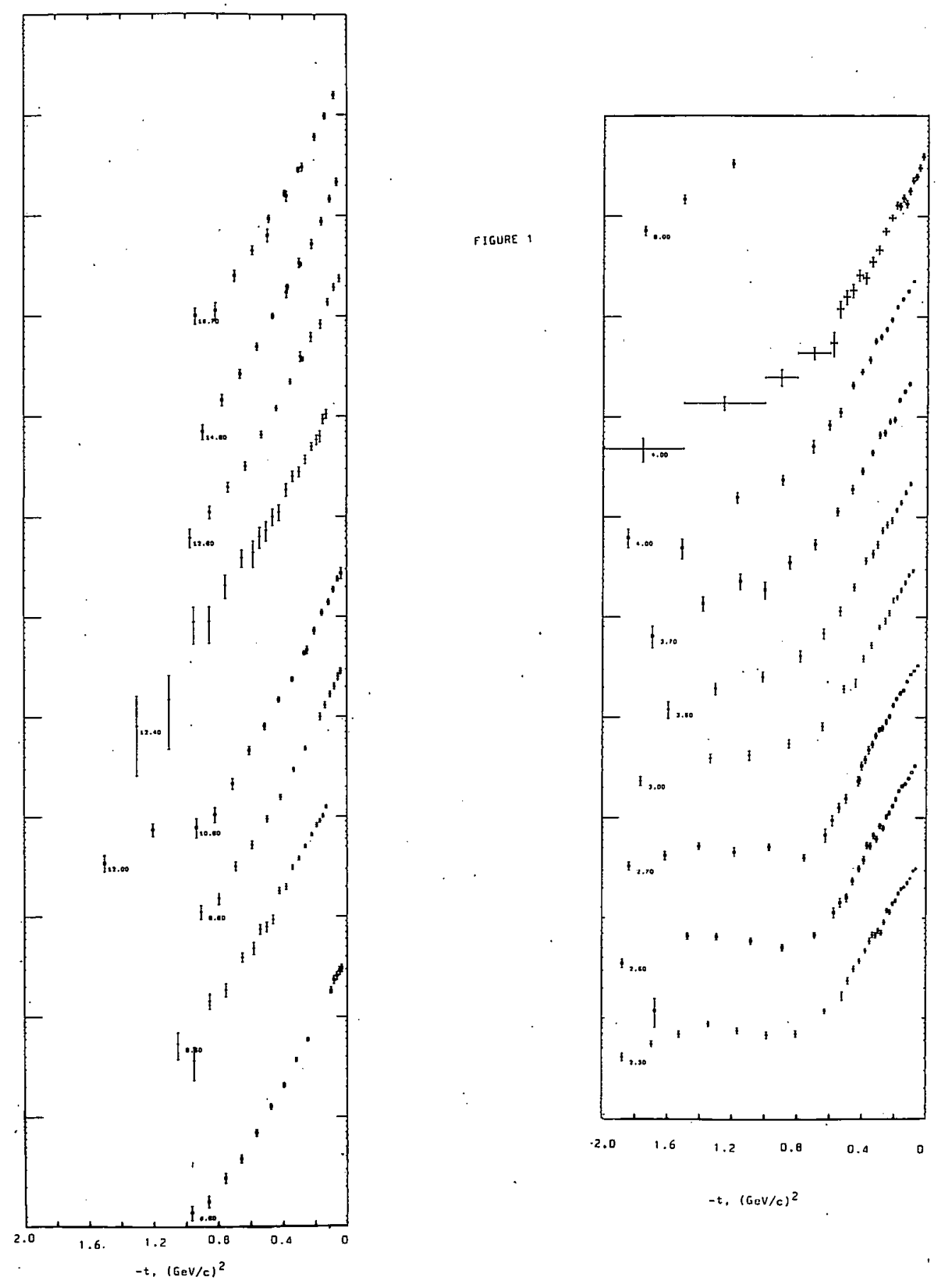

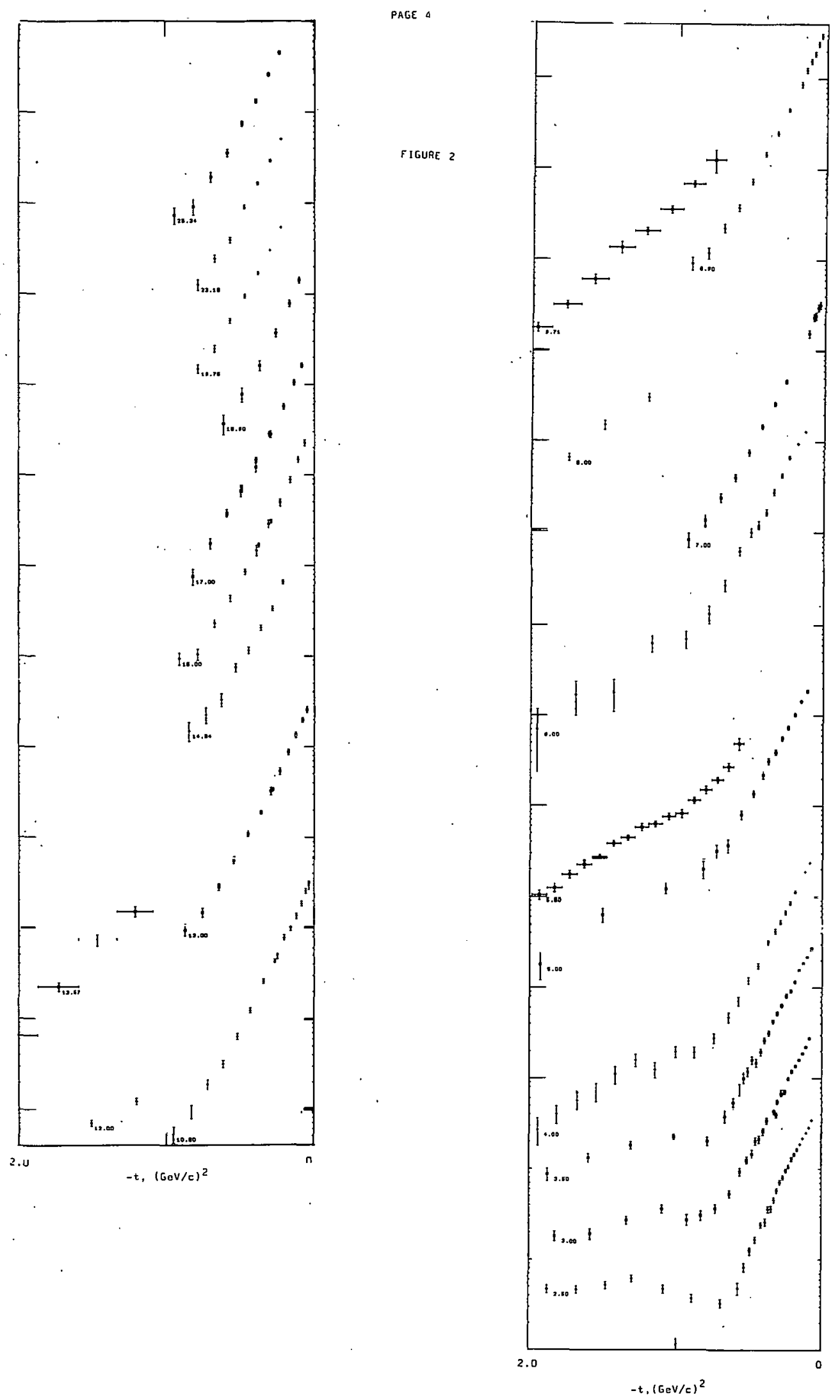


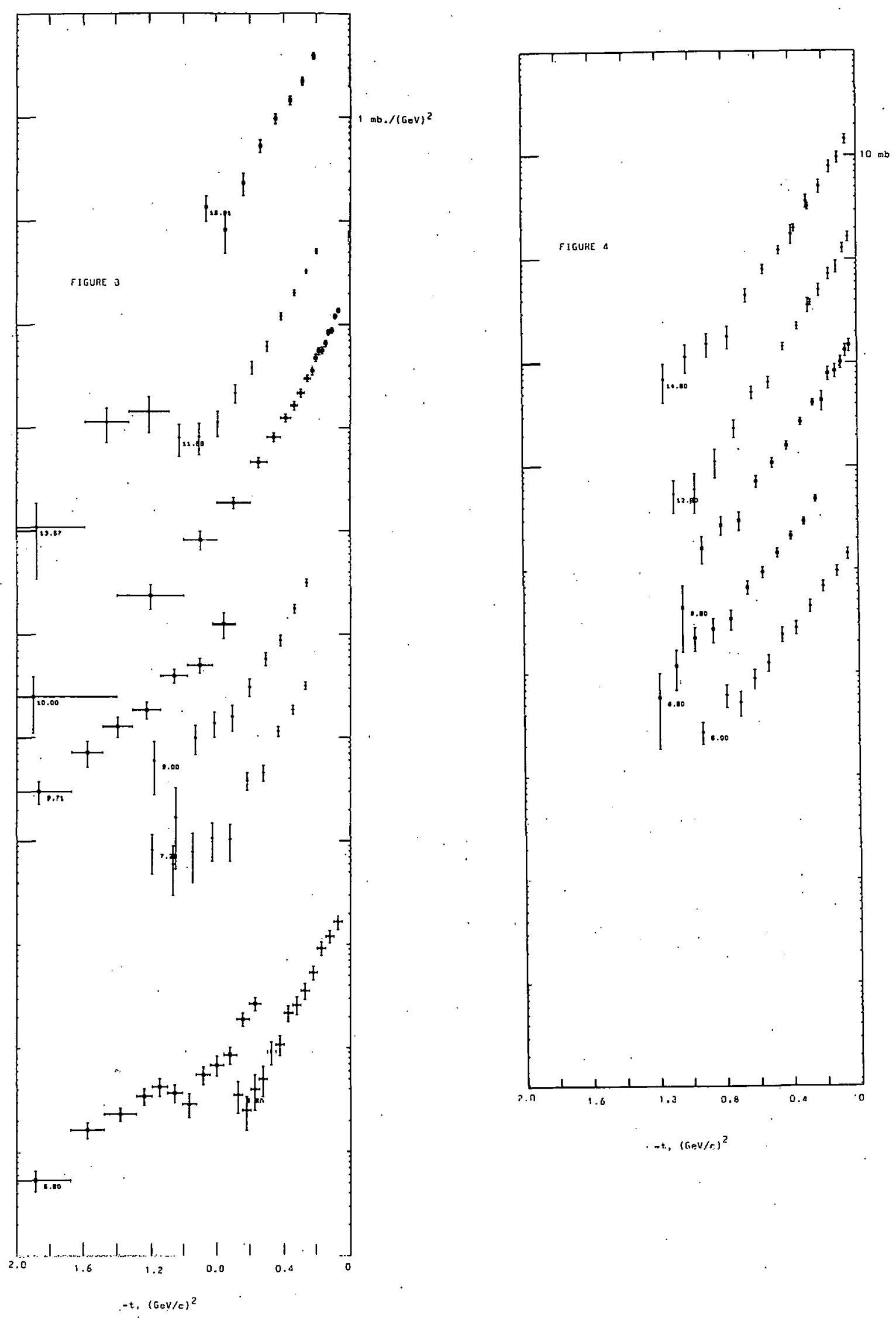


PAGE 6

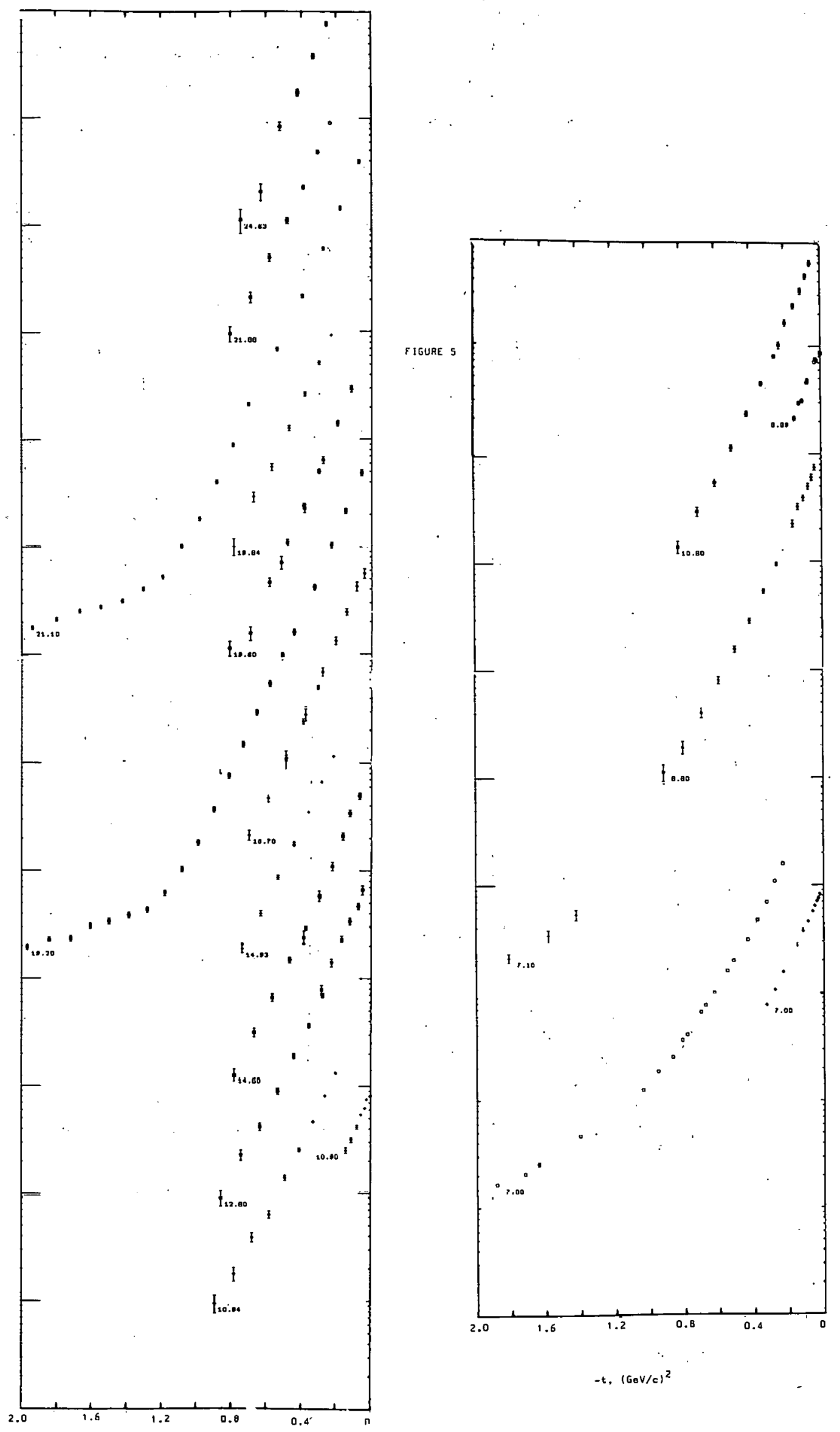

$-t,(G 0 v / c)^{2}$ 


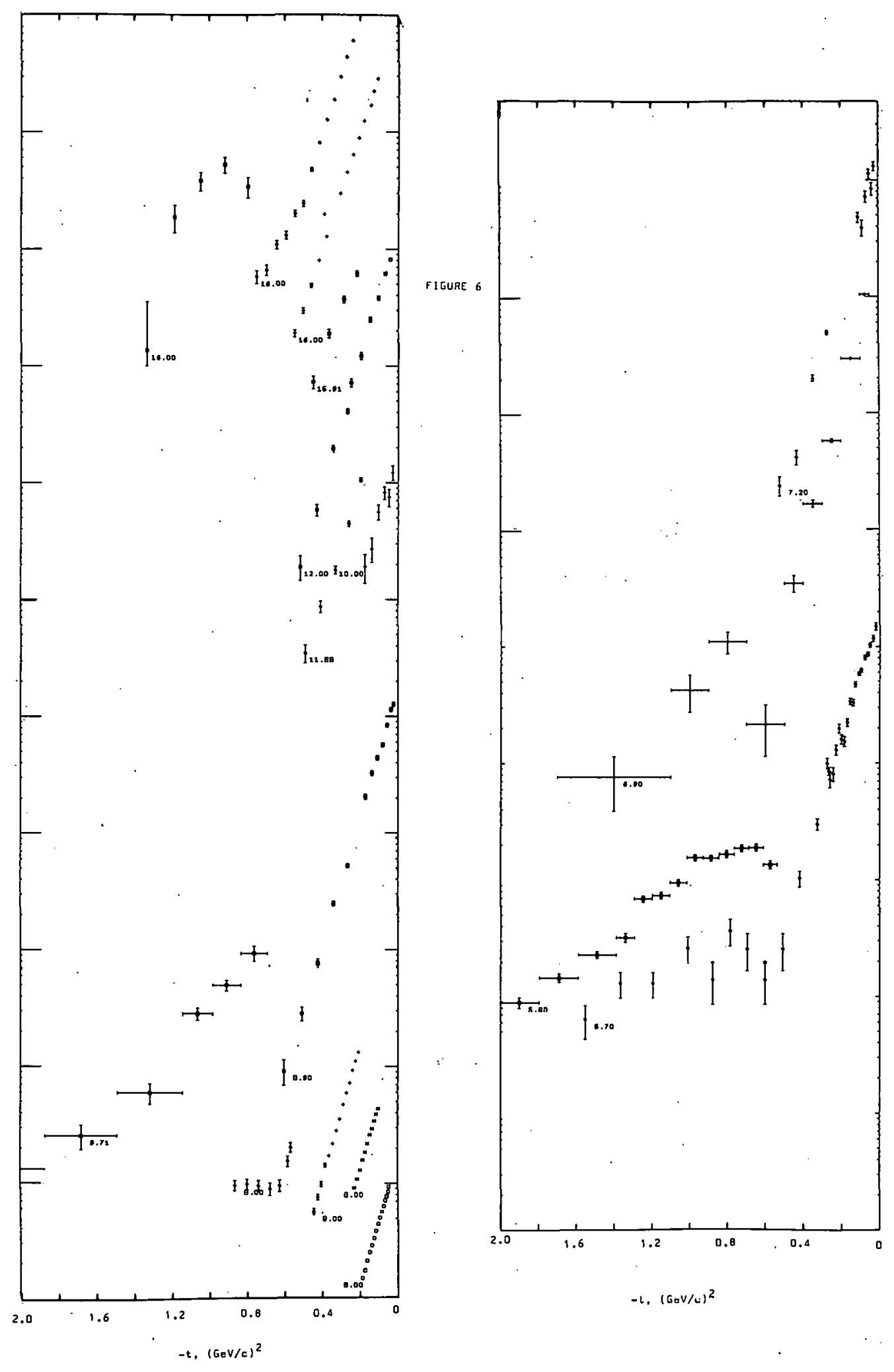


PAGE 8

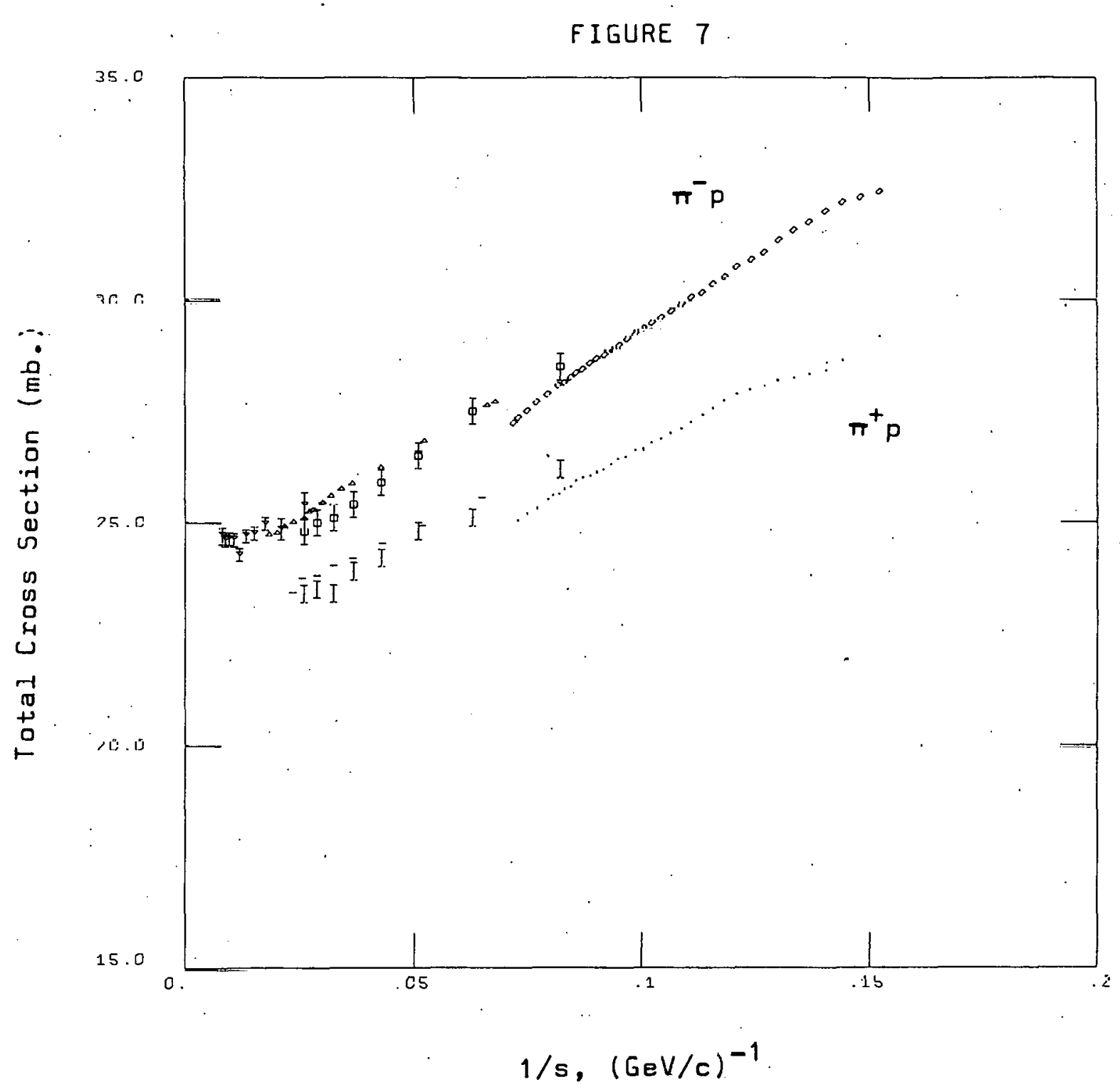


PAGE 9

FIGURE 8

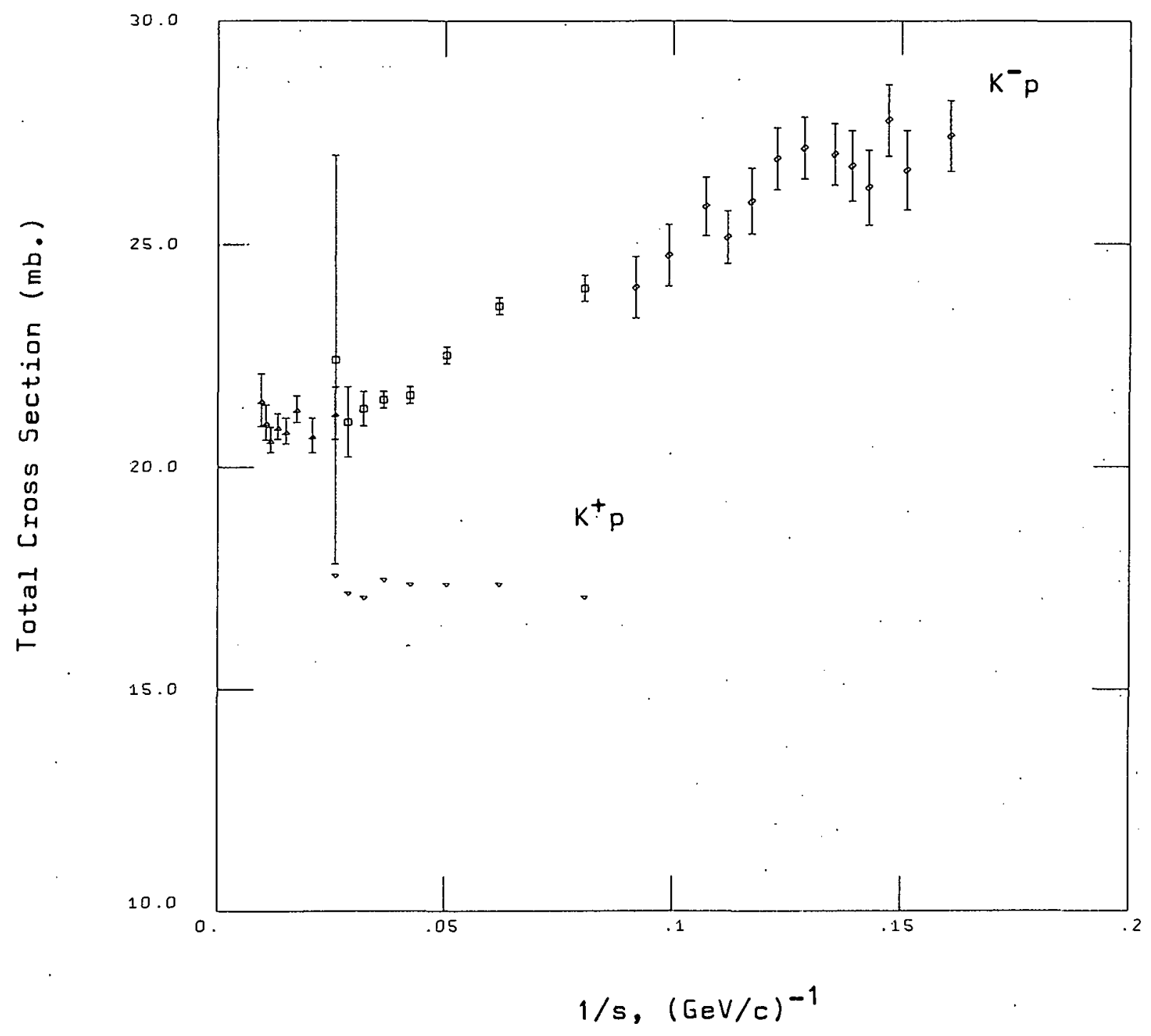


PAGE 10

FIGURE 9

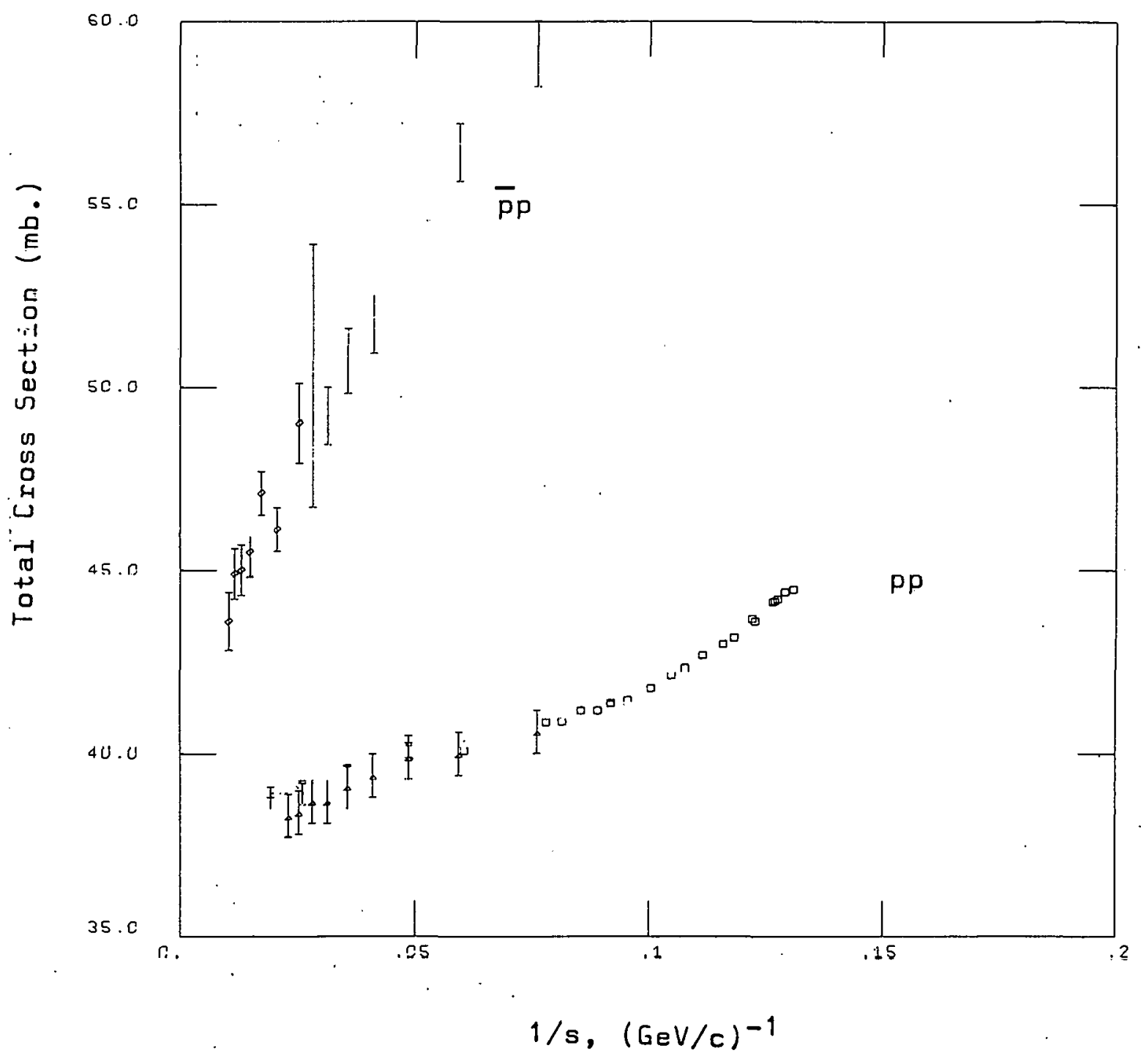



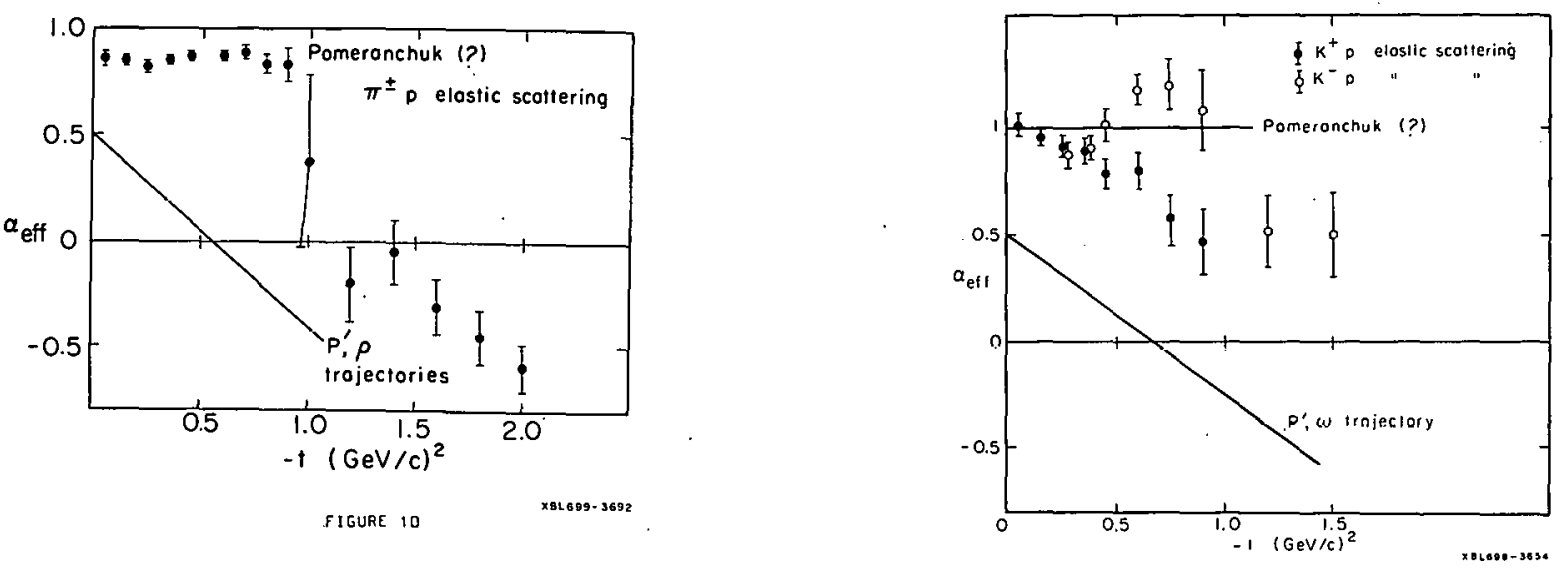

FI GUat 11
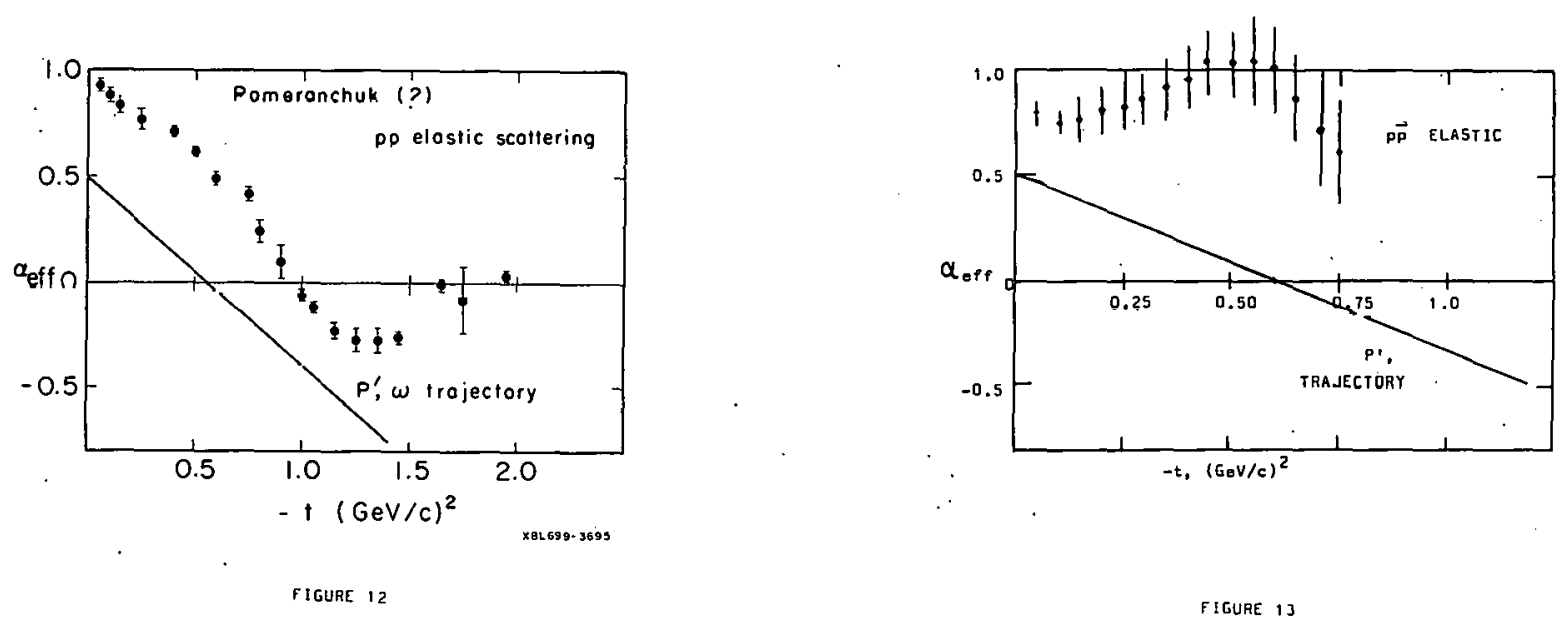


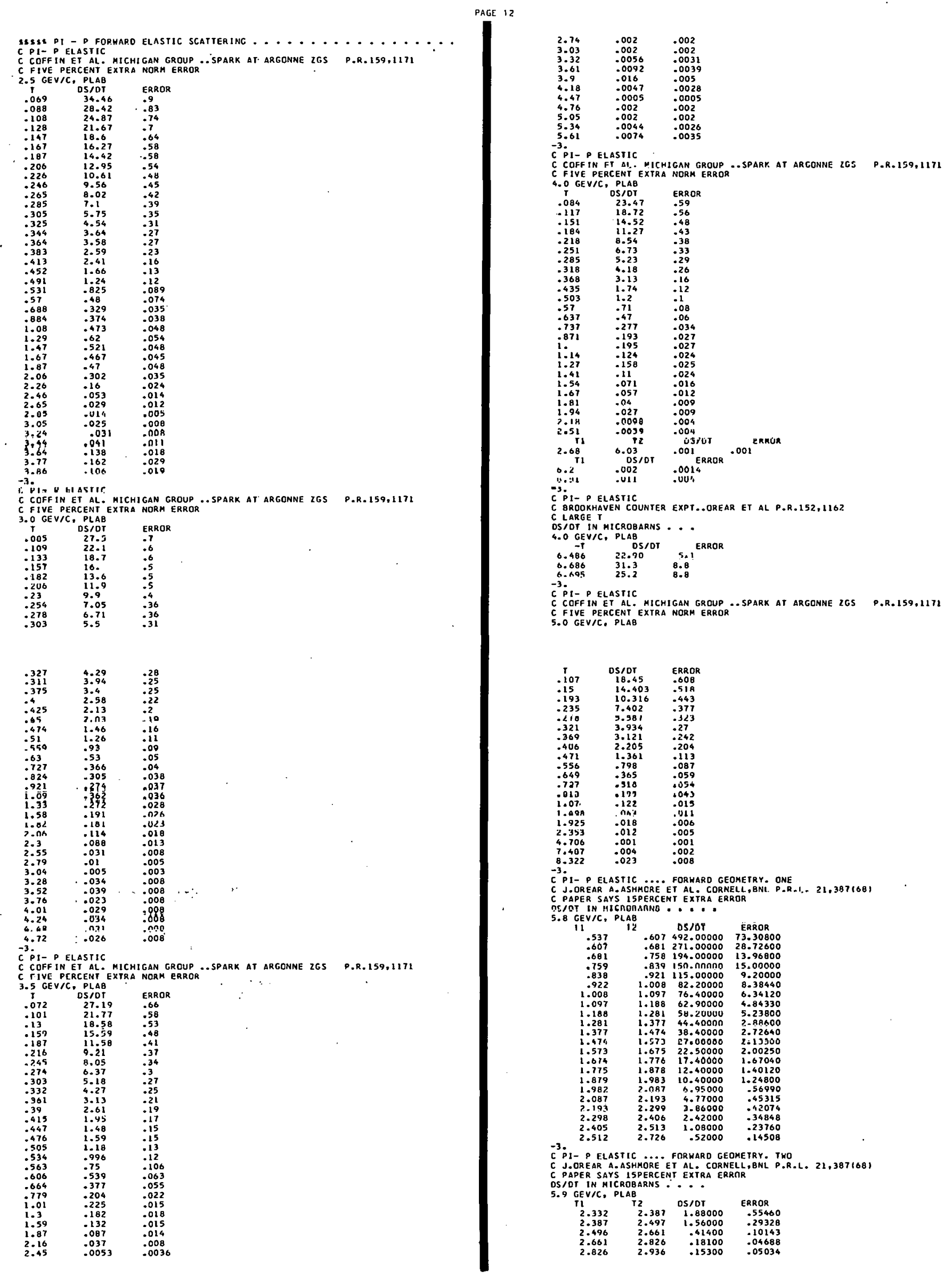




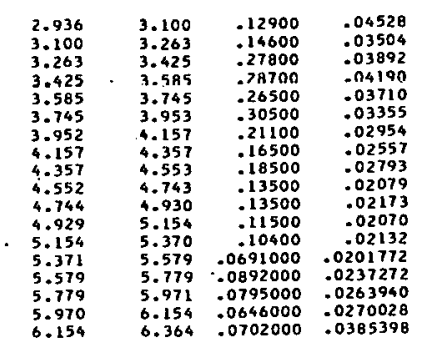

c3. PI- P ELASTIC

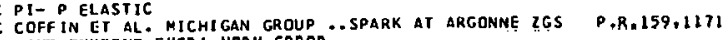
6. OGEV/C PLAB

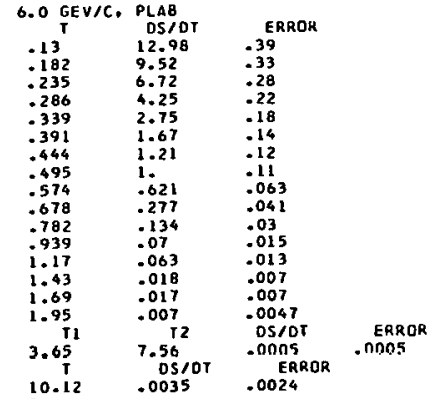

$c^{-3} \dot{p}_{1-}$ P ELASTIC

C LINDENBAUH P.R 11,42516313 PERCENT Relative error after ...APPROX.. . 25

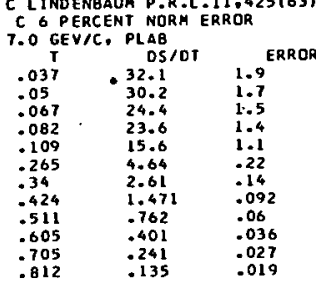

$.024 \quad 002 \quad .015$

CPI- E ELASTIC .... FORHARD GEDMETRY. TNO

c J.OREAR A.ASHMORE ET AL. CORNELL,

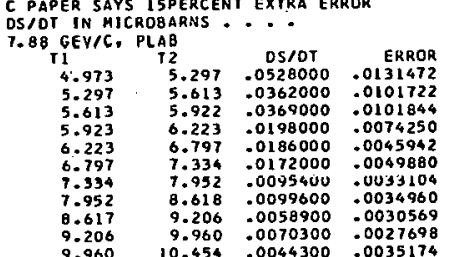

$c^{-3} \dot{p}_{1-P}$ elastic

$\begin{array}{rll}9.960 & .0070300 & .002769 \\ 10.454 & .0044300 & .0035174\end{array}$

CXPT. OREAR EI AL P.R.152,1162

CS LARG IN HICROBARNS.

\begin{tabular}{|c|c|c|}
\hline \multicolumn{3}{|c|}{$\begin{array}{l}\text { PLAB } \\
\text { US/OI }\end{array}$} \\
\hline & $\begin{array}{l}30.4 \\
15.1\end{array}$ & $\begin{array}{l}3.1 \\
1.8\end{array}$ \\
\hline & $\begin{array}{l}6.55 \\
2.71\end{array}$ & $\begin{array}{l}.58 \\
.65\end{array}$ \\
\hline 2.5 & $\begin{array}{l}.57 \\
.2\end{array}$ & $\begin{array}{l}.08 \\
.06\end{array}$ \\
\hline 2.72 & .047 & $\begin{array}{l}.019 \\
.019\end{array}$ \\
\hline 3. & $\begin{array}{r}-104 \\
-104\end{array}$ & .031 \\
\hline $\begin{array}{l}4.5 \\
5 .\end{array}$ & $\begin{array}{l}.086 \\
.083\end{array}$ & .041 \\
\hline 14.05 & $\begin{array}{l}2.03 \\
2.08\end{array}$ & $\begin{array}{l}: 03 \\
.78\end{array}$ \\
\hline $\begin{array}{l}14: 1 \\
14: 13 \\
1 \leqslant 1:\end{array}$ & 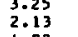 & $\begin{array}{r}.88 \\
.57\end{array}$ \\
\hline $\begin{array}{c}14.17 \\
14.16 \\
-3 .\end{array}$ & $\begin{array}{r}4.99 \\
3.35\end{array}$ & 1.94 \\
\hline \multirow{2}{*}{\multicolumn{3}{|c|}{ 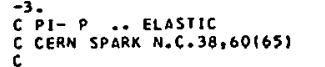 }} \\
\hline & & \\
\hline \multicolumn{3}{|c|}{$\begin{array}{l}\text { 8.5 GEV/C, PLAB } \\
\text { DS/DT }\end{array}$} \\
\hline $\begin{array}{l}.05 \\
.07\end{array}$ & 24.44 & 1.68 \\
\hline .09 & $\begin{array}{l}22.63 . \\
17.9\end{array}$ & $\begin{array}{l}1.69 \\
1.49\end{array}$ \\
\hline 1 & 14.36 & 1.34 \\
\hline & 10.94 & .29 \\
\hline & & .27 \\
\hline $\begin{array}{l}10 \\
.22\end{array}$ & $\begin{array}{l}8.01 \\
6.35\end{array}$ & $\begin{array}{l}.25 \\
.16\end{array}$ \\
\hline & & .12 \\
\hline & & 0 \\
\hline & & \\
\hline
\end{tabular}

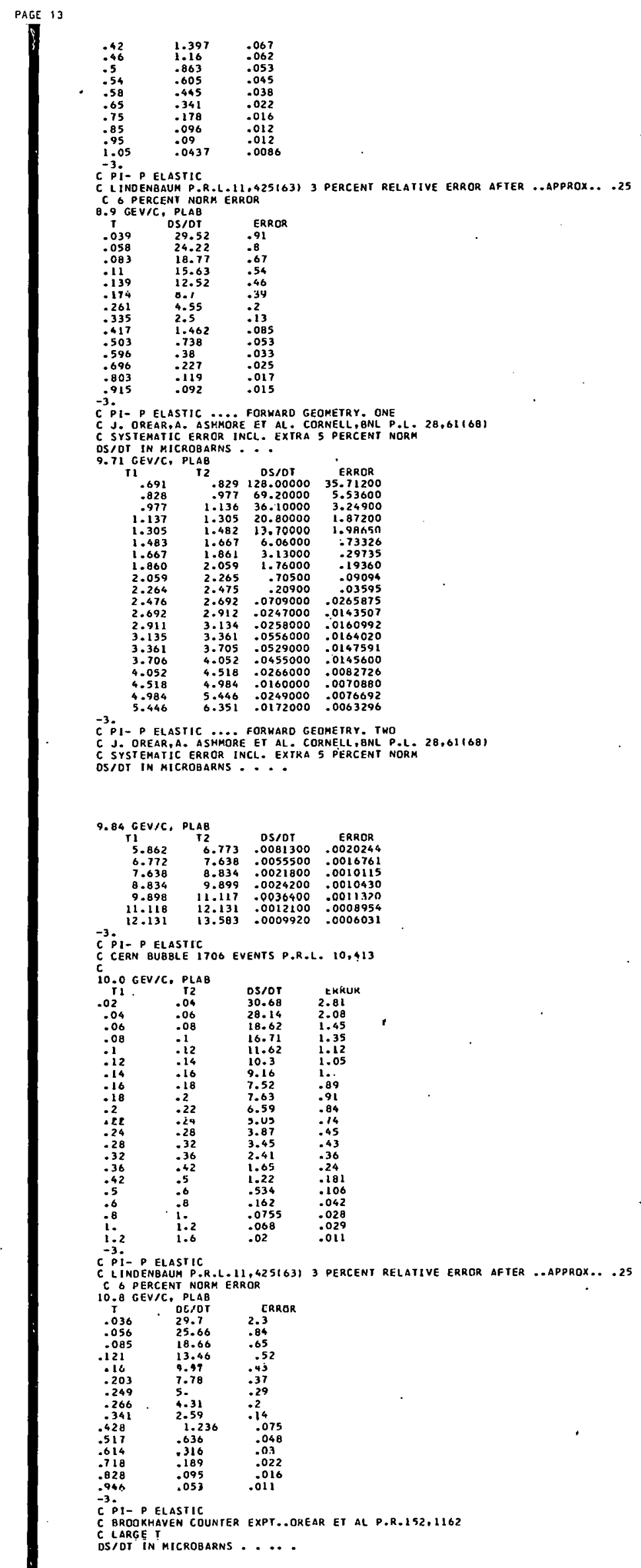



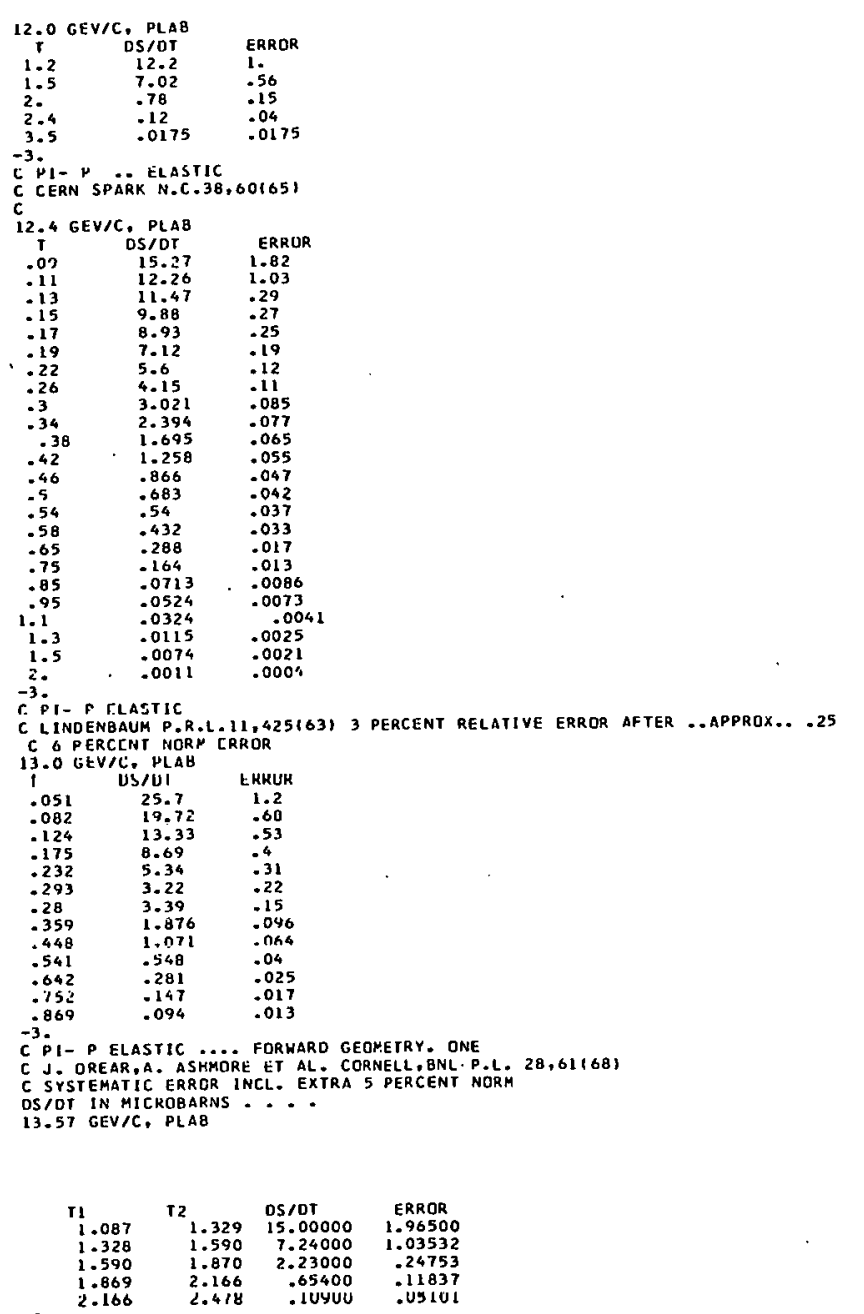

$c^{-3} \dot{p}_{1}$ - P ELASTIC

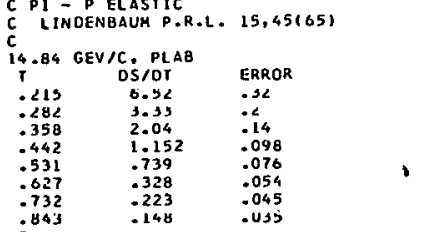

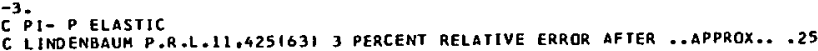
C O PERCENT MORM ERROR

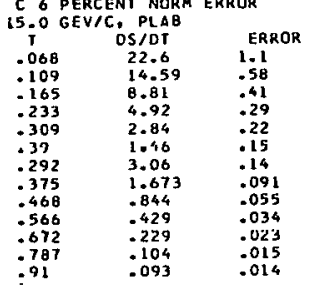

cípi-p ELASTIC

C LINDENBaum P.R.L.11,42516313 3 PerCent relative error after ..APprox.. .25 C 6 PERCENT NORH ERROR

\begin{tabular}{ccc}
17.0 GEV/C. PLAB & \\
T. DS 101 & ERROR \\
.086 & 16.33 & .58 \\
.006 & 16.33 & .58 \\
.139 & 10.44 & .4 \\
.209 & 5.68 & .26 \\
.297 & 2.79 & .16 \\
.349 & 1.21 & .107 \\
.495 & .052 & .07 \\
.304 & 2.81 & .15 \\
.391 & 1.462 & .091 \\
.488 & .695 & .054 \\
.59 & .377 & .036 \\
.701 & .174 & .023 \\
.821 & .075 & .015 \\
\hline 301 & &
\end{tabular}

C.3. pi- p .. ELASTIC

C CERN SPARK N.C.38,601651

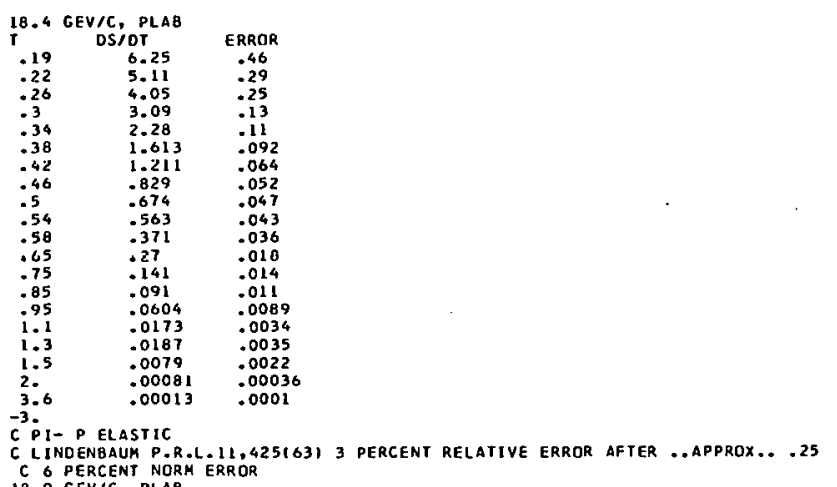

$C$
$1 A .9$
1

$\begin{array}{ccc}1 & \text { DS } / 01 & \text { ERRO } \\ .107 & 14.07 & .61 \\ .173 & 7.79 & .42 \\ .26 & 3.69 & .26 \\ .368 & 1.61 & .16 \\ .488 & .77 & .116\end{array}$

$\begin{array}{lll}.488 & .77 & .16 \\ .614 & .364 & .079\end{array}$

$c^{-3} \dot{P}_{I}=P$ ELASTIC

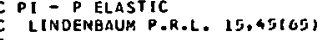

10,74 nEV/C, $D 1, \Delta R$

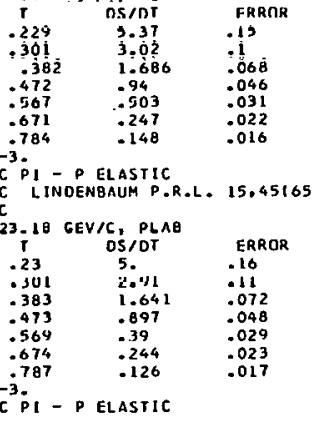

c. LINDENBAUM P.R.L. $15,45(65)$

25.34 GEV/C, PLAB

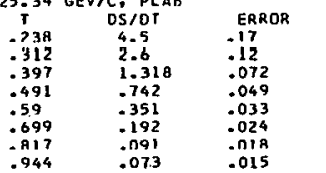

S3sp! - $\rho$ IUIAL LRUSS SECIIUNS

C PI-P ELASTIC
P.R. 144,110I BROOKHAVEN

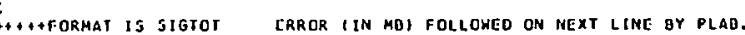

$32.299 \quad .015$

3.12

$32.174 \quad .015$

3. 22

$31.973 \quad .015$

$31.746 \quad .015$

ग.*

$31.569 \quad .015$

3.52

$31.334 \quad .015$

3.62

31.064 .015

$30.901 \quad .01$

3.82

$30.739 \quad .01$

3.93

$30.519 \quad .01$

4. 03

$30.363 \quad .01$

(*)

${ }_{4.23}^{30.17 \quad .01}$

** $30.058 \quad .01$

4.33

$29.902 \quad .01$ 


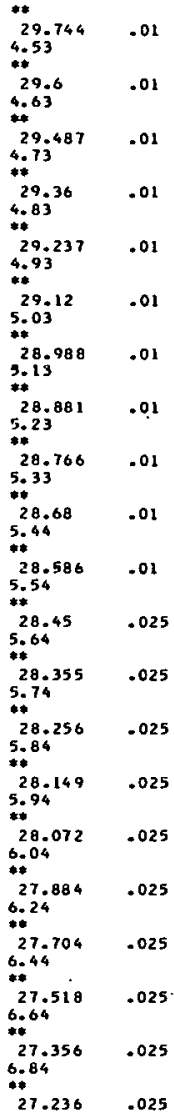

6.94

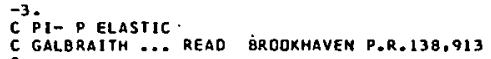

$*$

$\begin{array}{ll}28.5 & \\ 6 . & 223\end{array}$

$* 27.5 \quad .3$

8.8

10.

$25.9 \quad-3$

$25.4 \quad .3$

$25.1 \quad+3$

$16.12+3$

18.

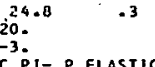

C LINOENBAUM P.R.L. 19,330IE622) 1671

$27.755 \quad .089$

**.671 .088

7.6

26.87

9.78

26.273
12.61

25.915

25.915
14.13

25.799
15.21

25.642

25.509
17.32

2.5.727

18.36 $\underset{710.64}{25.340}$

25.308

19.22

25.15
20.17

25.064
.22 .09

24.955
24.27

24.822
26.19

24.776
28.68

3.

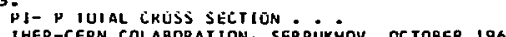

C IHEP-CERN COLABORATION SERPUKMOV OCC TOBER 1969
C NOW PUB. IN PL $30 B$. ADOITIONAL SYSTEMATIC ERROR AT LEAST 1

$25.38 \quad 0.300$

20.

24.85 $\quad 0.250$

25.

$24.97 \quad 0.150$

$34.75 \quad 0.150$

35.

$24.70 \quad 0.150$

$24.27 \quad 0.150$

45.

24.620 .150

$24.64 \quad 0.150$

55.

24.6

24.6
65.

35issp I- P ELASTIC ReAL/IMAGINARY at $T=0$

CPI- P ELASTIC

Th SOL OVIEV METHOO

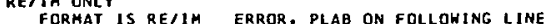

$-.12$ .07

3.40

$-.17$

$c^{-3} p_{1}$ - P ELASTIC

C LINDENBAUM P.R.L. 19,1931671

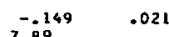

-.124
9.84

$\begin{array}{cc}-.167 & 02\end{array}$

$\begin{array}{rr}-.137 \\ 11.89 & .017\end{array}$

$\begin{array}{rl}-131 & .025\end{array}$

-.143
19.44

$-.144 \quad .022$

-.112
18.19

-.117
20.15

$\begin{array}{rl}-.132 & .017\end{array}$

$-.123 \quad .026$

-.125
24.24

$\because 2128 . \quad 026$

SSISSP 1- P ELASTIC POLARIZATION

C PI- P ElaSTIC polarization

C ESIERLLNG ET AL CHICAGO ILLINOIS VIENNA BB

S.15 GEV TC PLAB

\begin{tabular}{|c|c|c|c|}
\hline .15 & $\therefore$ & & \\
\hline & & $\mathbf{P}$ & ERROR \\
\hline & $\begin{array}{l}.254 \\
.314\end{array}$ & $=07$ & $\begin{array}{r}.024 \\
.023\end{array}$ \\
\hline & $\begin{array}{r}.38 \\
.44\end{array}$ & -.042 & $\begin{array}{l}.025 \\
.03\end{array}$ \\
\hline .532 & .532 & .04 & 03 \\
\hline 7 & .723 & $\begin{array}{r}0.048 \\
-048\end{array}$ & .060 \\
\hline
\end{tabular}




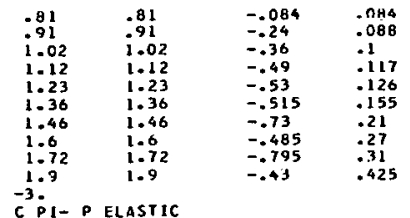

C CERN POLARIZATION P.L. 24,771671 P.L,21,114 G.0 r.FV/r, PLAB

\begin{tabular}{|c|c|c|c|}
\hline 1 & & $\mathbf{P}^{\prime}$ & ERROR \\
\hline .1309 & $\begin{array}{r}109 \\
.136\end{array}$ & $\begin{array}{l}=13 \\
=-166\end{array}$ & $\begin{array}{l}.034 \\
.018\end{array}$ \\
\hline .168 & .166 & -.153 & .014 \\
\hline 198 & .198 & -.170 & .014 \\
\hline 233 & .233 & -.115 & .015 \\
\hline .269 & .269 & -.146 & .017 \\
\hline .311 & .311 & -.093 & .02 \\
\hline 354 & .354 & -.08 & .022 \\
\hline & & -.058 & .027 \\
\hline $\begin{array}{l}447 \\
497\end{array}$ & -497 & -.038 & $\begin{array}{r}.037 \\
.042\end{array}$ \\
\hline 549 & .549 & (03) & .076 \\
\hline .604 & .604 & .004 & .074 \\
\hline 661 & .661 & & .082 \\
\hline 72 & .72 & .054 & .121 \\
\hline 78 & .78 & .117 & .266 \\
\hline
\end{tabular}

C. PI- P ELASTIC

CERN POLARILATION P.L.24,771671 P.L.21,114 EET RELATIVE ERROR

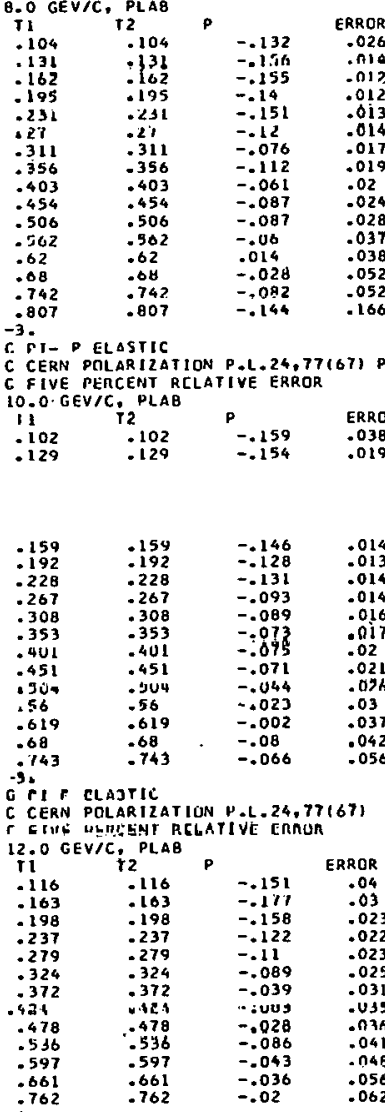

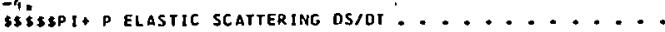

COFFIN ET AL. PICHIGAN GROUP .. SPARX AT ARGONNE $26 S$ P.R.159,117 C FIVE PERCENT EXTRA NORM ERROR

\begin{tabular}{|c|c|c|}
\hline $\begin{array}{l}2.3 \mathrm{G} \\
\mathrm{T}\end{array}$ & 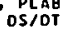 & ERROR \\
\hline .062 & 31.2 & 1. \\
\hline $\begin{array}{l}.00 \\
.098\end{array}$ & 29.4 & $\because 9$ \\
\hline .116 & 22.4 & $\because 8$ \\
\hline .134 & 20.4 & .7 \\
\hline .152 & 19.6 & .7 \\
\hline .169 & 17,5 & .7 \\
\hline $\begin{array}{r}-187 \\
\end{array}$ & 15. & .6 \\
\hline .305 & 13.9 & .6 \\
\hline .223 & 11.5 & .6 \\
\hline - 241 & 11.9 & .6 \\
\hline - 259 & 9.1 & .5 \\
\hline 218 & 7.1 & 46 \\
\hline 294 & 7.45 & -45 \\
\hline .312 & 6.7 & -4 \\
\hline .33 & 6.8 & .4 \\
\hline 34 & 5.9 & -4 \\
\hline 3 & 4.7 & .25 \\
\hline & $\begin{array}{l}3.7 \\
3.1\end{array}$ & $?$ \\
\hline 81 & 2.35 & .18 \\
\hline 517 & 1.66 &.-16 \\
\hline .624 & 1.17 & .08 \\
\hline
\end{tabular}

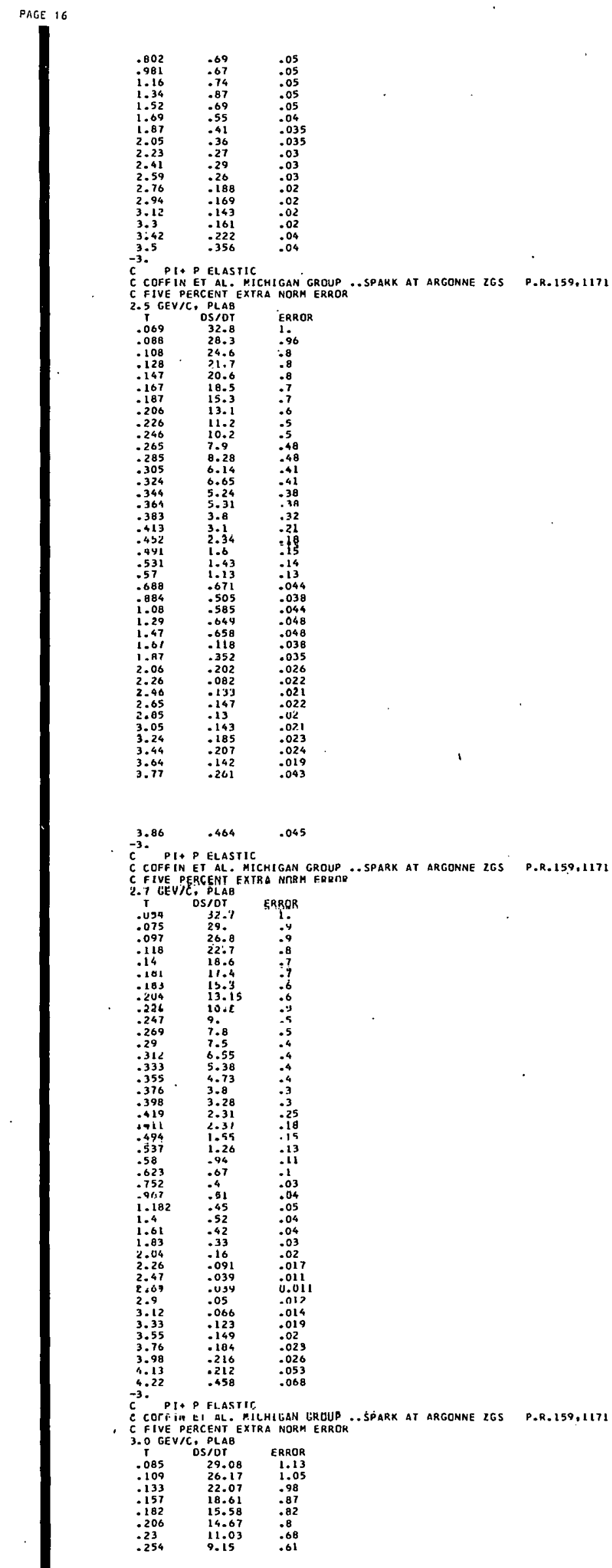



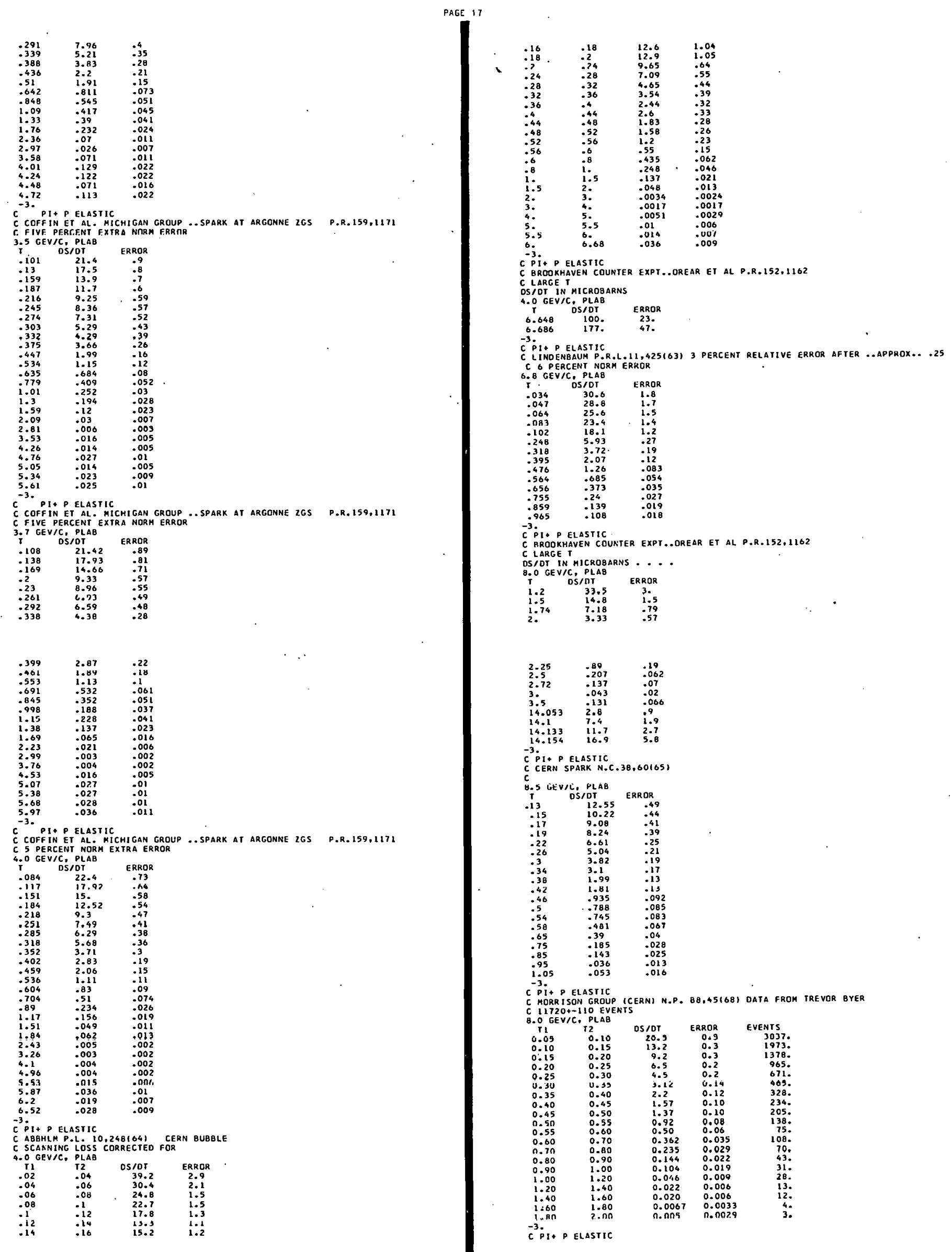
C LINDENBAUM P.R.L. 11,42516313 PERCENT RELATIVE ERRDR AFter ..APPROX.. . 25 C. PERCENT NORM ERRO

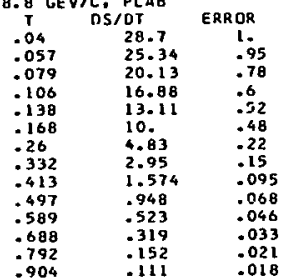

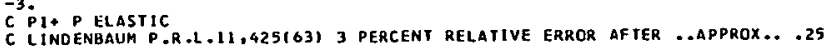
C C O PERCENT NORM ERROR

C 6 PERCENT NORH
10.8 GEVIC, PLAB

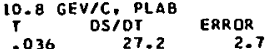

$\begin{array}{ll}.036 & 27.2 \\ .054 & 23.9 \\ .084 & 18.7\end{array}$

$-116 \quad 14$.

$-157$

.249
.260

.341

.424

.606

.817
.933

14.98
10.98
7.23

4.64
4.31

2.36
1.478

.804
.46
.105

.214
.105
.078

CHA. P ELASTIC

Chanue

CUUNIER EXPT...UREAH EI AL M.R.152,118:

plobarns...

$\begin{array}{ccc}\text { L2.0 GEV/C, PLAB } & \\ 1 & \text { DSOT } & \text { ERROR } \\ 1.2 & 7.42 & 1.11 \\ 1.5 & 3.45 & .62 \\ 2.5 & .51 & .23 \\ 2.4 & .3 & .12\end{array}$

$c^{-3} \dot{p}_{\text {It }}$ P ELASTIC

C CERN SPARK N.C. 38,601651

C. 2 OEV/C, PLAB

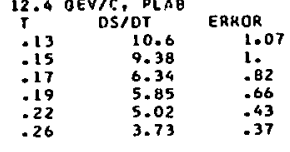

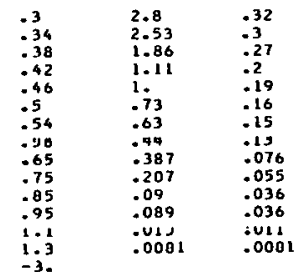

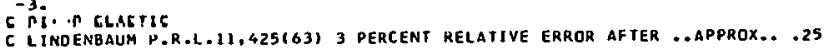
$C$ O PERCENT NORH ERROR

12.8 GEV C C P PAB

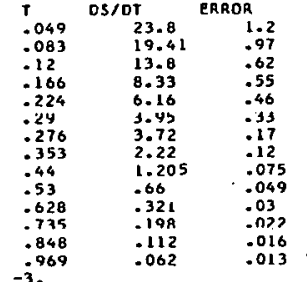

$c \dot{p}_{1+} p_{\text {ELASTIC }}$

CERE RELATIVE ERROR AFTER ...APPROX... 25

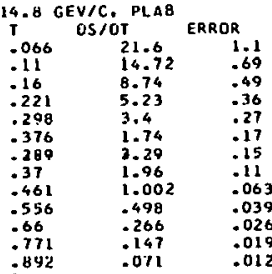

$c^{-3} c_{1}$. P ELASTIC

.R.L.11,4251631 3 PERCENT RELATIVE ERROR AFTER ..APPROX.. .25

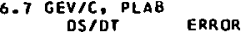

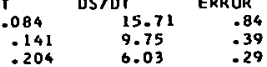

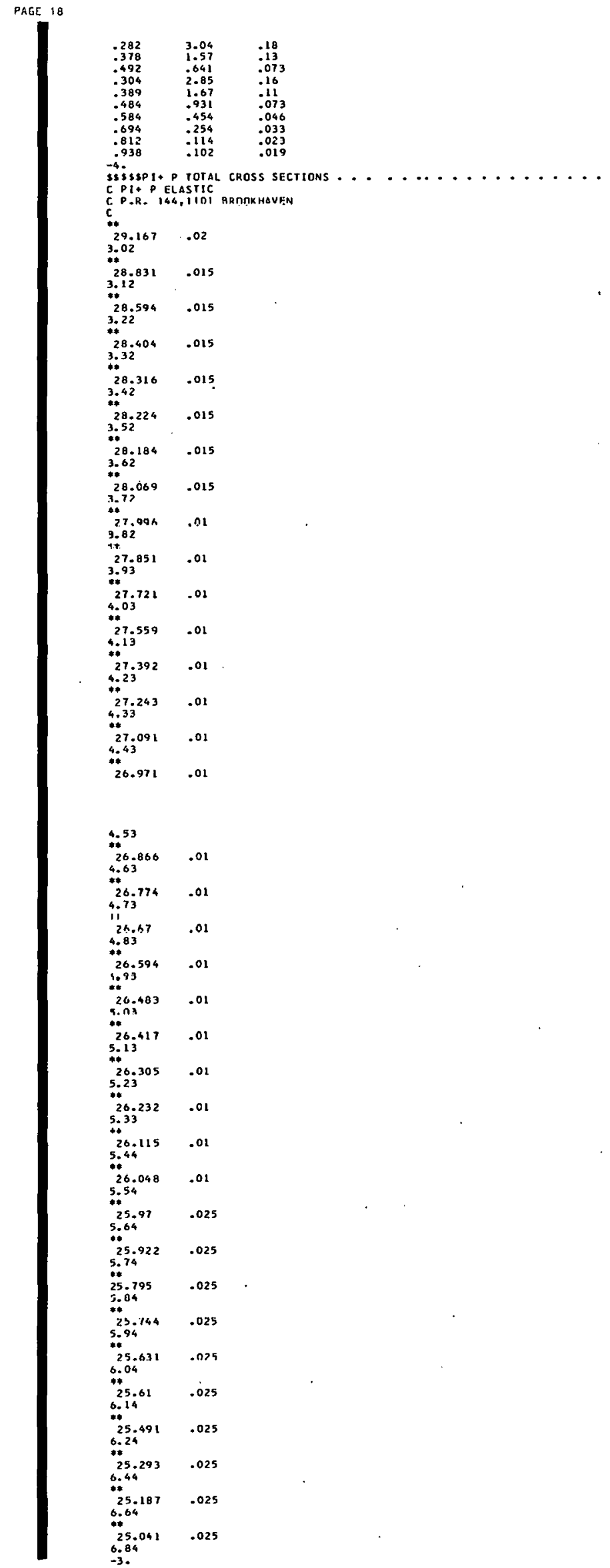



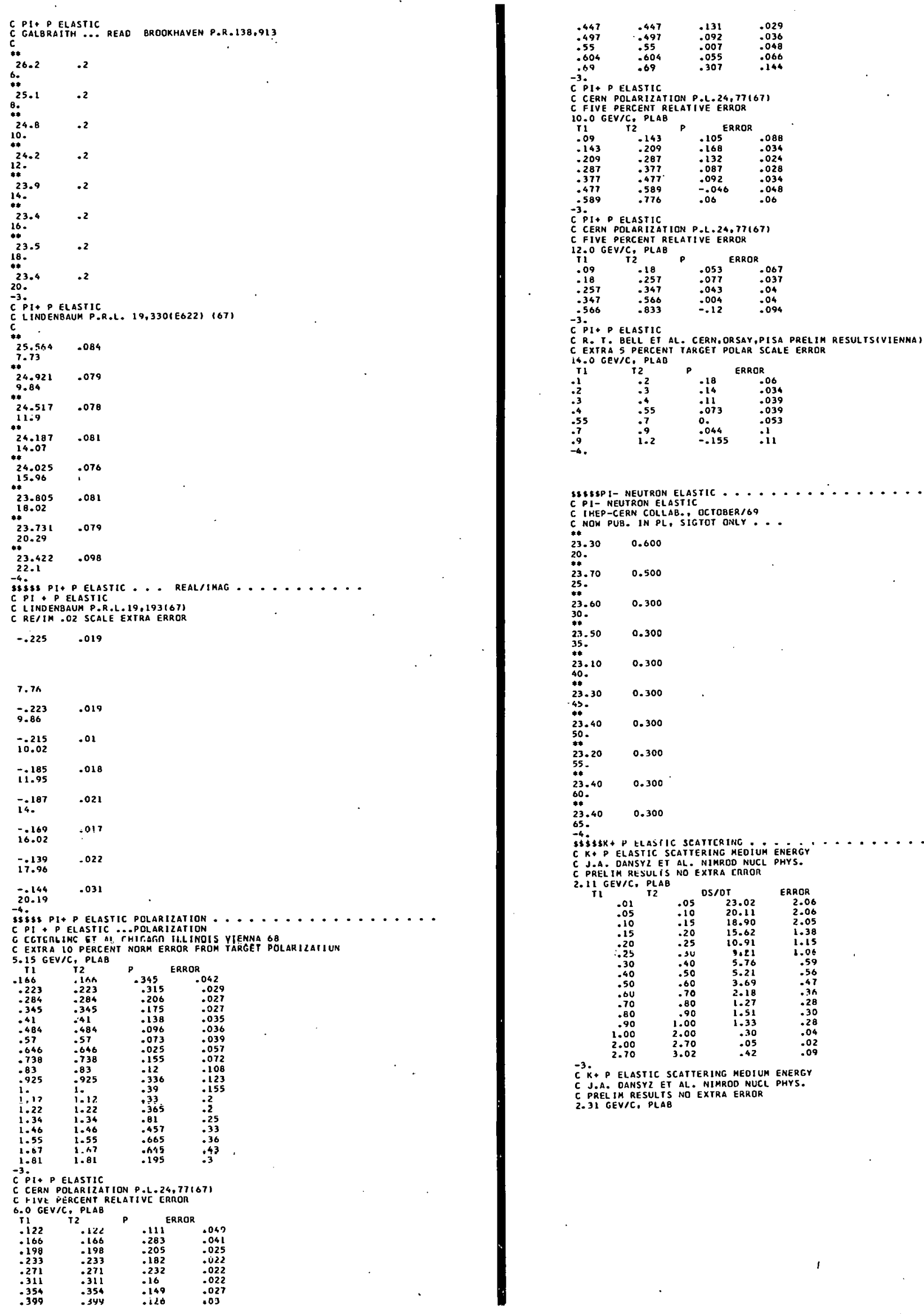

SSBISP I- NEUTRON ELASTIC . . .

IHEP-CERN COLLAB, OC TOBER/69

C NOW PUB. IN PL, SIGTOT ONLY...

23. $30 \quad 0.600$

20.

$23.70 \quad 0.500$

**.60 0.300

30.60

$23.50 \quad 0.300$

35.

$23.10 \quad 0.300$

40.

$23.30 \quad 0.300$

43.

$23.40 \quad 0.300$

50.

$23.20 \quad 0.300$

40

$23.40 \quad 0.300$

(**

23.40

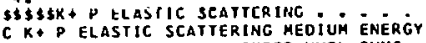

MROD NUCL PHYS. C PRELIIM RESUL IS NO EXIRA CRROR

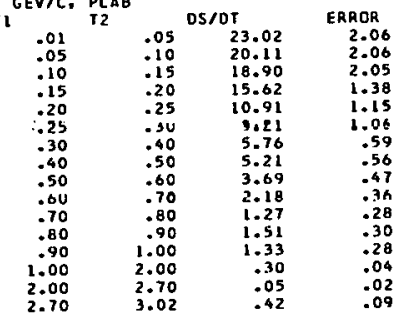

$c^{-3} \dot{K}+p$ elastic scattering meOIUM ENERGY

C J.A. DANSYZ ET AL. NIMROD NUCL PHYS.

C PREL IM RESULIS NO EXIRA ERROR

2.31 GEV/C, PLAB 


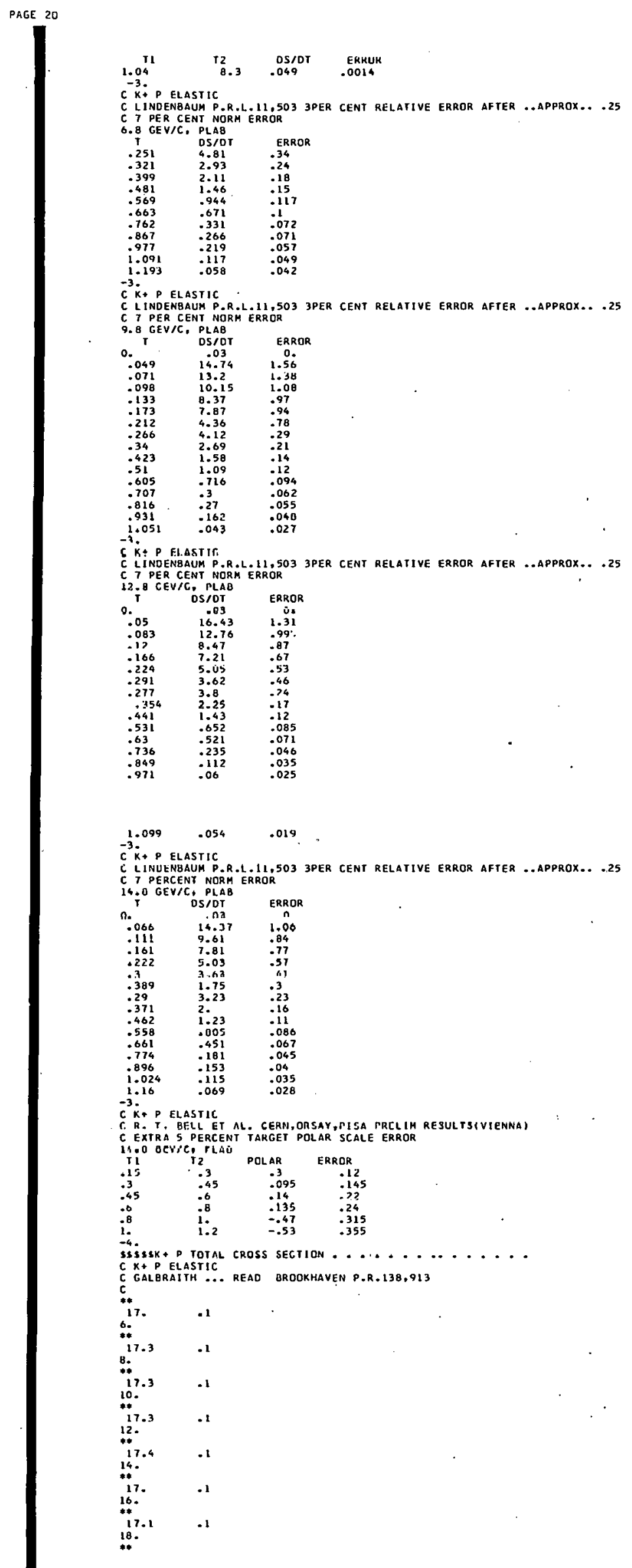




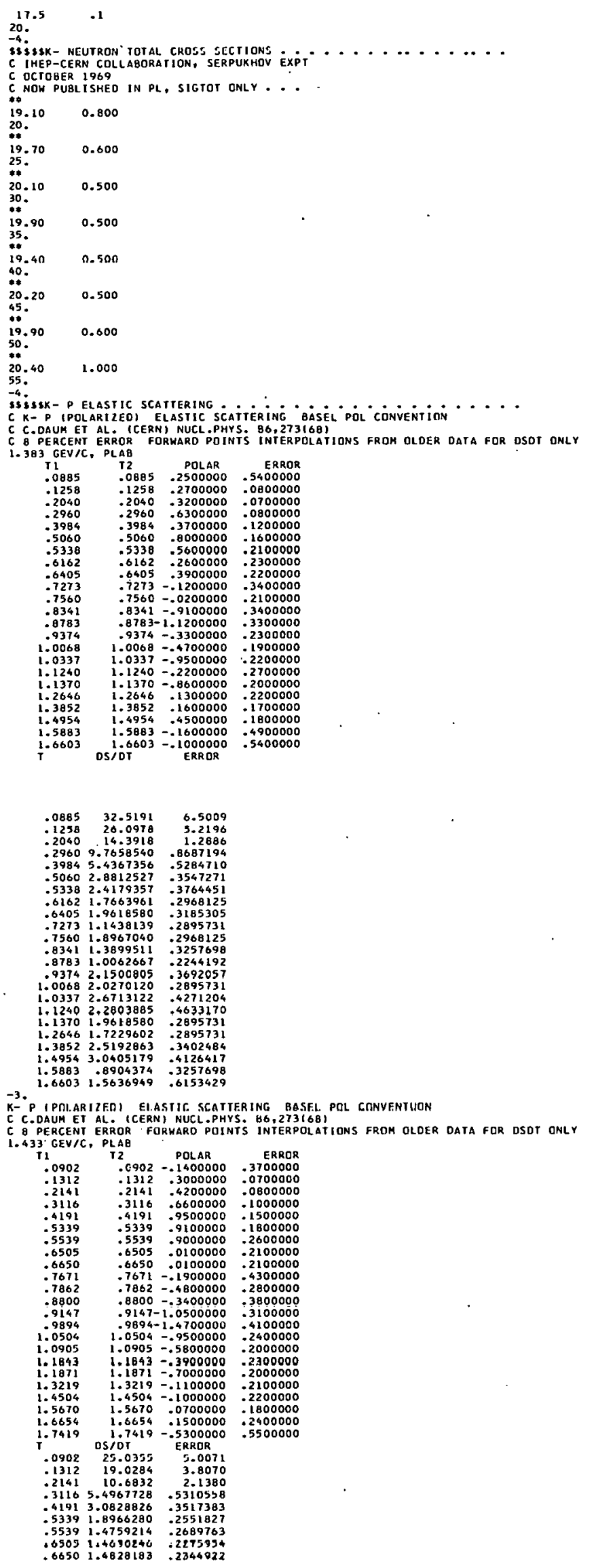




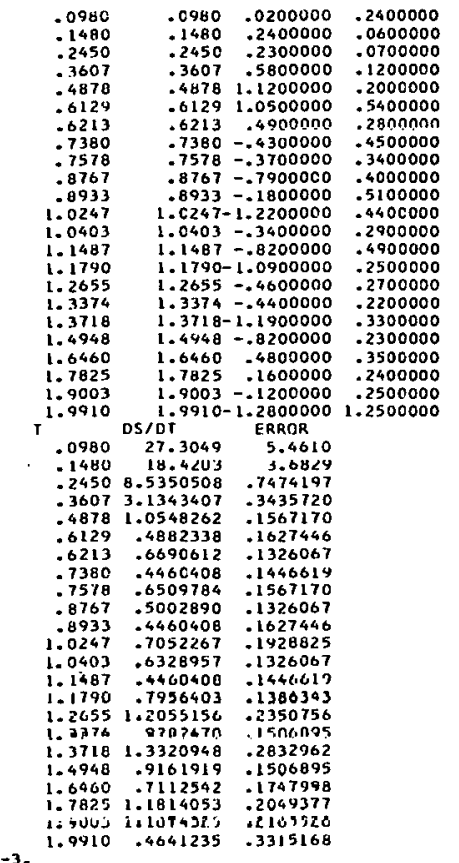

C K-P IPOL. I ELASTIC, HASEL CONVENTION

C C.DAUT ET AL. (CERN) NUCL. PHYS. PA.PTJ(GB) 1.63\%, GEV/C, PLAE

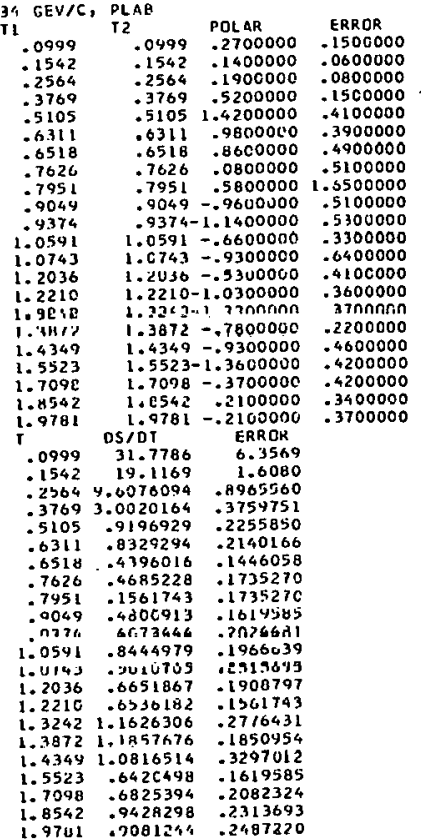

-3- P (PCLARILED) Elastic SCATtering Rasel pol conventuon

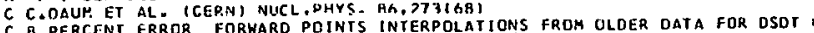

L. OU4 GEV T, PLA

$\begin{array}{llll}.1029 & 12 & \text { POLAR } & \text { ERRDR } \\ .1594 & .1029 & .3300000 & .2000000 \\ .1594 & .2600000 & .0600000 \\ .2667 & .2667 & .3600000 & .0700000 \\ .3945 & .3945 & .4600000 & .1400000 \\ .5340 & .5346 & -.0300000 & .2800000 \\ .6499 & .5499 & .1200000 & .3700000\end{array}$

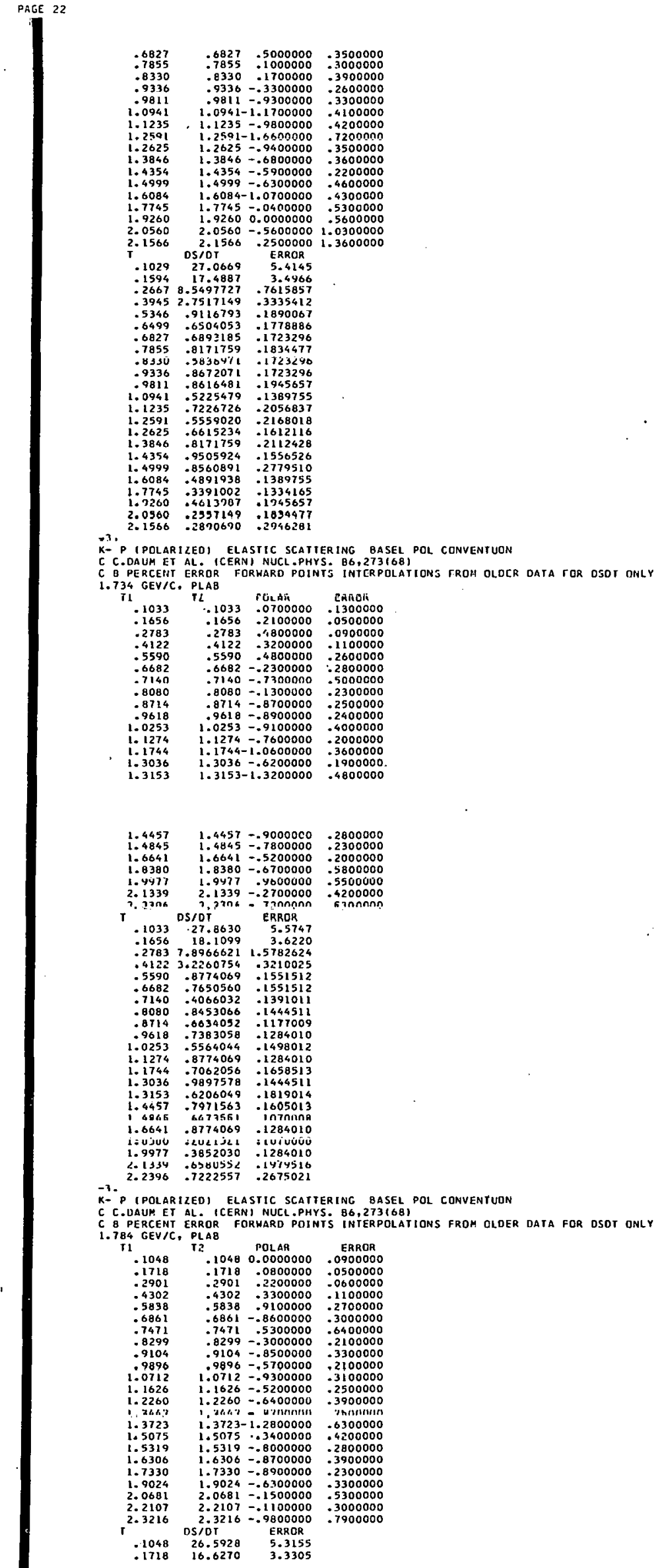




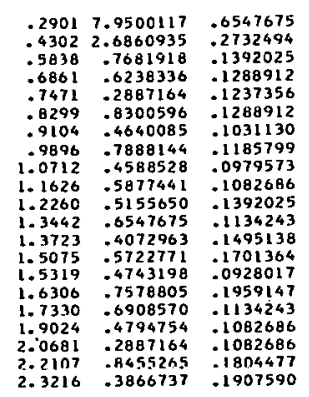

K- P (POLARIZED) ELASTIC SCATIER ING BASEL POL CONVENTUON C 8 PERCENT ERROR FORWARD POINTS INTERPOLATICNS FROM OLDER DAIA FOR DSDT ONLY 1.884 GEV/C, PLAB

\begin{tabular}{|c|c|c|c|}
\hline & & & \\
\hline & & POL & \\
\hline & .1098 & .0800000 & 00000 \\
\hline .184 & .1844 & .2000000 & 0000 \\
\hline .313 & .313 & .3800000 & .0700000 \\
\hline .466 & .4668 & $\begin{array}{r}3900000 \\
\end{array}$ & -12000000 \\
\hline .0355 & .6355 & 1.2800000 & .4300000 \\
\hline $\begin{array}{r}.8120 \\
.8735\end{array}$ & .8735 & $\therefore .9700000$ & .4000000 \\
\hline 98 & & -.9300000 & .4000000 \\
\hline 1.0435 & 1.0435 & -.3800000 & .2600000 \\
\hline 1.16 & & -.4700000 & .3100000 \\
\hline 1.22 & 1.2279 & -.3700000 & .2200000 \\
\hline 1.3312 & 1.3312 & $=.5100000$ & $\begin{array}{r}3200000 \\
.4600000\end{array}$ \\
\hline $\begin{array}{l}1.4240 \\
1.4881\end{array}$ & & & .7400000 \\
\hline 1.6280 & 1.6280 & -.3900000 & .3100000 \\
\hline 1.6332 & 1.6332 & -.8600000 & .5100000 \\
\hline 1.8320 & 1.8320 & -.8700000 & $\begin{array}{r}2900000 \\
3400000\end{array}$ \\
\hline $\begin{array}{l}2.0294 \\
2.2112\end{array}$ & $\begin{array}{l}2.0294 \\
2.2112\end{array}$ & $\because 7300000$ & .3900000 \\
\hline & 2.3681 & .8400000 & .6300000 \\
\hline & OS\% $10 T$ & ERROR & \\
\hline-1098 & 29.0946 & 5.8189 & \\
\hline $\begin{array}{r}.1844 \\
.3138\end{array}$ & $\begin{array}{r}16.8753 \\
+.1979675\end{array}$ & $\begin{array}{r}1.3070 \\
-6390719\end{array}$ & \\
\hline .4668 & 2.4361612 & .2642778 & \\
\hline .6355 & .52 & .13 & \\
\hline .81 & .2786 & .134 & \\
\hline .87 & .4660 & .129 & \\
\hline .9899 & .5621 & .14 & \\
\hline 1.0435 & .67 & .124 & \\
\hline 1.1638 & .52 & .115 & \\
\hline 1.227 & .888 & .144 & \\
\hline & & & \\
\hline & .3267435 & .0961010 & \\
\hline & .4084294 & .1681768 & \\
\hline
\end{tabular}

\section{$1.6280 \quad .5237506 \quad .1153212$ \\ $\begin{array}{lll}1.6332 & .5862163 & .1922021 \\ 1.8320 & .6102416 & .1153212\end{array}$ \\ $\begin{array}{lll}2.0294 & .4516749 & .1105162 \\ 2.2112 & .4228446 & .1105162\end{array}$}

-3-p ipolarizeo, elastic scattehing basel pol conventuon

C C.DAUM ET AL. (CERN) NUCL.PHYS. B6, $273(68)$ I 1.934 GEY/C, PLAB

\begin{tabular}{|c|c|c|c|}
\hline ? & $T_{2}$ & POLAR & \\
\hline $\begin{array}{l}-11 \\
.19\end{array}$ & -1172 & - ?hnonann & - ก90nnnnก \\
\hline $\begin{array}{r}.1907 \\
.3259\end{array}$ & $\begin{array}{l}1907 \\
.3259\end{array}$ & $\begin{array}{r}.2300000 \\
.2900000\end{array}$ & $\begin{array}{l}.0500000 \\
.0400000\end{array}$ \\
\hline .48 & .4855 & .5200000 & .1000000 \\
\hline .6612 & .0612 & .5400000 & .2500000 \\
\hline $\begin{array}{r}.8452 \\
1.0318\end{array}$ & $\begin{array}{r}.8452 \\
.0318\end{array}$ & $\begin{array}{l}=-1200000 \\
-5400000\end{array}$ & $\begin{array}{r}-3700000 \\
3000000\end{array}$ \\
\hline 2116 & 1.2116 & $\begin{array}{l}-.5400000 \\
-.3200000\end{array}$ & .2300000 \\
\hline 1.3847 & 1.3847 & -.5100000 & .2800000 \\
\hline 1.5470 & 1.5470 & -.6100000 & .3700000 \\
\hline $\begin{array}{l}1.6741 \\
1.6971\end{array}$ & $\begin{array}{l}1.6741 \\
1.4071\end{array}$ & $\begin{array}{r}-.3300000 \\
.42 \text { 23nnกn }\end{array}$ & $\begin{array}{l}.2600000 \\
.4 \Delta \text {. }\end{array}$ \\
\hline 8323 & 1.8323 & -.6600000 & .4400000 \\
\hline & 1.8864 & -.4100000 & .2900000 \\
\hline $\begin{array}{l}2.0919 \\
2.7812\end{array}$ & 2.0919 & -.0100000 & .1800000 \\
\hline $\begin{array}{l}2.2812 \\
2.5733\end{array}$ & 2.2812 & -.5900000 & 1.2000000 \\
\hline & DS & ERRQR & \\
\hline & 23. & 4.7719 & \\
\hline & 13.85 & 2.7693 & \\
\hline & .1612367 & .4507 & \\
\hline 4855 & 1.5008141 & .144 & \\
\hline 12 & .3949511 & .0696 & \\
\hline 8452 & .2509101 & .0650 & \\
\hline .8 & .3345468 & .0696 & \\
\hline & .4925272 & .0789 & \\
\hline & .3066679 & .0604043 & \\
\hline 6 & .3345460 & .0836367 & \\
\hline & 4 & .05575 & \\
\hline & .302 & .0929297 & \\
\hline & .2648495 & .0789902 & \\
\hline & .2602031 & .0557 & \\
\hline & $.41,1$ & $n 1,04$ & \\
\hline & 0743437 & .0604043 & \\
\hline & 11113 & .0929297 & \\
\hline
\end{tabular}

X- $p$, polarized elastic scatiering basel pol conventudon

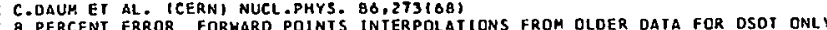
.984 GEV/C, PLAB

$\begin{array}{cccc}\text { B4 GEV/C, PLAB } & \text { VULAK } & \text { EKKUK } \\ \text { T1 } & 12 & .0900 \\ .1146 & .1146 & .1400 & .0900 \\ .1970 & .1970 & .1900000 & .0500000 \\ .3381 & .3381 & .2700000 & .0700000 \\ .5043 & .5043 & .4700000 & .1100000 \\ .6873 & .6873 & .9600000 & .3400000 \\ .7530 & .7530 & -.5600000 & .6000000 \\ .8767 & .8787 & -.2100000 & .4800000 \\ .7164 & .7164 & -.1700000 & .2400000 \\ 1.0715 & 1.0715 & -.7700000 & .2900000\end{array}$

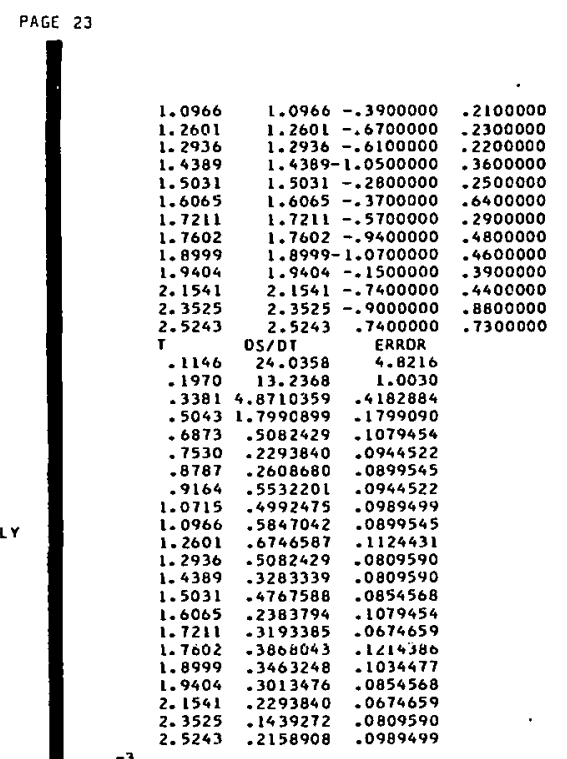

-3-P (PQLARILED) ELASIIC SCATIERING BASEL POL CONVENTUON

C C.DAUM ET AL. (CERN) NUCL. PHYS, B6,273168) 2.034 GEV/C. PLAB

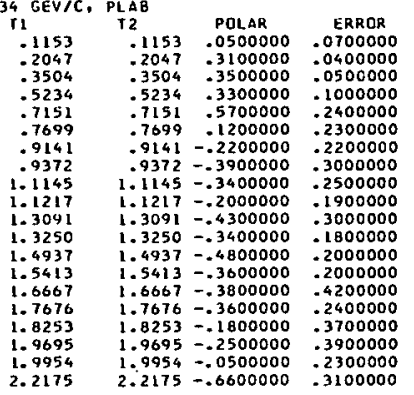

$2.4236 \quad 2.4236-1.1200000 \quad .6900000$

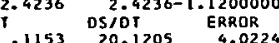

$.2047 \quad 10.6028 \quad 2.1136$

.35na 3.757163 A . THA

.5234
.7151

$.7699 \quad .3747806 \quad .0653687$

$.0141 .3617068 \quad .0610108$

$\begin{array}{rrr}.9372 & .2527590 & .0566529\end{array}$

$\begin{array}{lll}1.1145 & .3442752 & .065368 \\ 1.1217 & .3834964 & .0566529\end{array}$

$\begin{array}{lll}1.1217 & .3834964 & .056635 \\ 1.3091 & .2963381 & .0653687\end{array}$

$\begin{array}{lll}1.3250 & .3922122 & .0522950 \\ 1.4937 & .3878543 & .0566529 \\ 1.5413 & .3666648 & .0566529\end{array}$

$\begin{array}{lll}1.5413 & -3660648 & .0566529 \\ 1.0667 & .2222536 & .0697260\end{array}$

$\begin{array}{lll}1.6667 & .2222536 & .0697266 \\ 1.7676 & .2309694 & .0435791\end{array}$

I. $8253 \quad .2832644 \quad .0784424$

$\begin{array}{lll}1.9695 & .2353273 & .0697266 \\ 1.9954 & .2745486 & .0479371\end{array}$

$2.2175 \quad .1917482 \quad .0435791$

-3. p (polarized) elastic scattering basel pol conventuon

C C.DAUM ET AL. (CERN) NUCL.PHYS. 86,2731681 A PERCENT ERROR

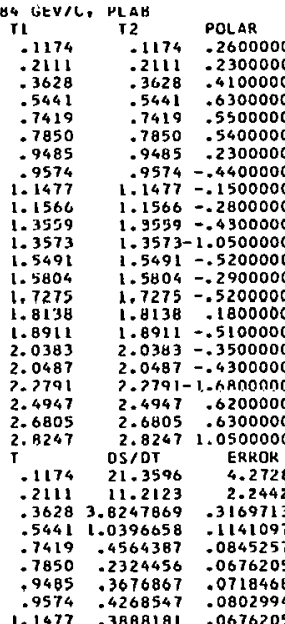




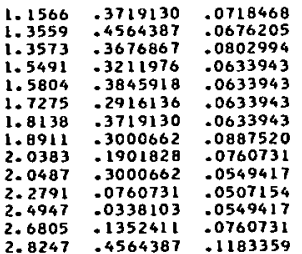

K- P (POLARIZED) Elastic SCATtering Basel pOL conventuon

C C.DAUM ET AL. (CERN) NUCL.PHYS. BG, $273(68)$ ) 2. 134 GEV/C. PLAB

\begin{tabular}{|c|c|c|}
\hline & & \\
\hline 19 & 1195 & \\
\hline & .2175 & $\begin{array}{r}3000000 \\
.2000000\end{array}$ \\
\hline & .3753 & .4300000 \\
\hline 3 & .5637 & .6500000 \\
\hline & .7704 & .7500000 \\
\hline .8164 & .8164 & .40 \\
\hline $\begin{array}{l}.9772 \\
.9849\end{array}$ & .9772 & -.46 \\
\hline $\begin{array}{r}-9849 \\
-1733\end{array}$ & $\begin{array}{r}.9849 \\
1.1733\end{array}$ & $\begin{array}{l}-.09 \\
-.48\end{array}$ \\
\hline 1993 & $\begin{array}{l}1.1993 \\
\end{array}$ & -.5700000 \\
\hline 3877 & 1.3877 & -.410 \\
\hline 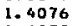 & & \\
\hline sos & & \\
\hline 617 & & $-.5 c$ \\
\hline & i. & -.2 \\
\hline & i. & -.18 \\
\hline & & \\
\hline & 2 & -.38 \\
\hline 105 & 2 & -.30 \\
\hline & Tחו.. & $\begin{array}{c}7300000 \\
\text { seng }\end{array}$ \\
\hline .118 & & 4.6395 \\
\hline .21 & 11. & \\
\hline & 5 & .965 \\
\hline & & \\
\hline & & \\
\hline $\begin{array}{l}.0164 \\
.9772\end{array}$ & $\begin{array}{r}5086669 \\
4\end{array}$ & -1066560 \\
\hline .9849 & .3732859 & .0902474 \\
\hline 17 & 10 & .08 \\
\hline .19 & .5250 & .0984514 \\
\hline 13 & .57 & .0820430 \\
\hline & .697 & \\
\hline & .6399 & .1107581 \\
\hline & & .07 \\
\hline & .4348 & .0861452 \\
\hline 05 & .3691 & .0730387 \\
\hline .9575 & .7547960 & .1353710 \\
\hline 10 & .2 & .0615323 \\
\hline .1091 & .1886990 & .1066560 \\
\hline 3419 & .0820430 & .0574301 \\
\hline
\end{tabular}

$2.3419 \quad .0820430 \quad .057430$

K- P (POLARI2ED) ELASTIC SCATTERING BASEL POL CONVENTUON

C C. DAUM ET AL. (CERN) NUCL. PHYS. B6, 273 (68) 2. LTS GEVIC, PLA

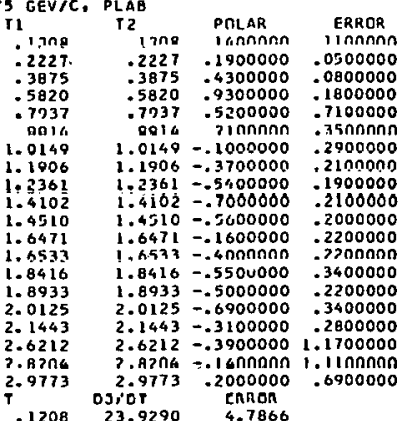

.1208 $23.9290 \quad \begin{aligned} & \text { Cnnon } \\ & 2.7860\end{aligned}$

$.2227 \quad 11.2070 \quad .0092$

$.5820 \quad .9012443 \quad .1241714$

$.9914 \quad .2523484 \quad .064088$

.0045585 .084116

$1.1906 \quad-5367410 \quad .0841161$

$1.4102 \quad .5247244 \quad .0801106$

$1.4510-7450286-1121548$

$\begin{array}{lll}1.4671 & .6785417 & .0245946 \\ 1.6533 & .5207189 & .084116\end{array}$

1.8416 .3324590 .000110

$2.0125 \quad .5046968 \quad .1201859$

$\begin{array}{lll}2.1443 & .3364645 & .0680940 \\ 2.6212 & .0921272 & .076105\end{array}$

$2.8204, .1121548$.088121

K- P (polarizeo) elastic scattering basel pol conventuon

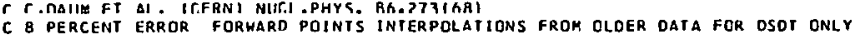
C 8 PERCENT ERROR FORHARD POINTS INTER
2. $2 B 4$ CEV $/ C$. PLAB

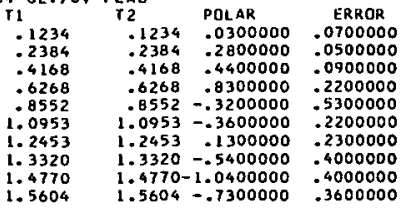

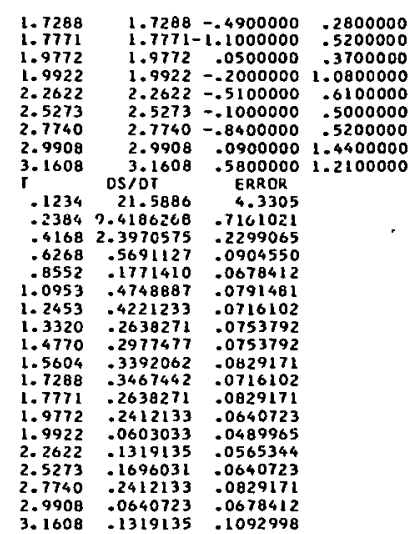

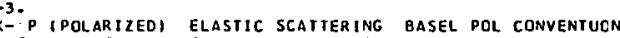

C C. OAUM ET AL ICERN) NUCL.PHYS. BG, 273168) 2.325 GEV/C, PLAB

\begin{tabular}{|c|c|c|c|}
\hline & & POLAH & ERKOR \\
\hline 124 & .1244 & .1800000 & .0900000 \\
\hline .427 & $\begin{array}{l}.2454 \\
.4278\end{array}$ & .32000000 & $\begin{array}{r}.0600000 \\
.1100000\end{array}$ \\
\hline & th? & .9900000 & .5400000 \\
\hline $\begin{array}{l}.8810 \\
.1263\end{array}$ & .8811 & $\begin{array}{r}-4000000 \\
-11200000\end{array}$ & $\begin{array}{r}-4300000 \\
4400000\end{array}$ \\
\hline 1.3702 & $\begin{array}{l}1.3702 \\
130\end{array}$ & -.3100000 & .2200000 \\
\hline 1.6054 & 1.6054 &,- 9500000 & .20000000 \\
\hline $\begin{array}{l}7553 \\
8200\end{array}$ & 1.7553 & -.3700000 & .2400000 \\
\hline $\begin{array}{l}.8260 \\
.0263\end{array}$ & 1.02600 & -1.0000000 & $\begin{array}{r}84000000 \\
400000\end{array}$ \\
\hline $\begin{array}{l}0203 \\
.3024\end{array}$ & 2.3024 & $=.4400000$ & .05000000 \\
\hline 57 & $2.575 \mathrm{i}$ & 1.1200000 & .7900000 \\
\hline & 2.8307 & -.8700000 & 1.6900000 \\
\hline & & .1900000 & .4000000 \\
\hline & OS/OT & ERROR & \\
\hline-24 & 9.17 i6514 & $\begin{array}{r}3.4657 \\
.0960187\end{array}$ & \\
\hline .42 & 1.7 & .24 & \\
\hline .04 & & .07 & \\
\hline .881 & .1880304 & .0553 & . \\
\hline 1.1265 & .1585355 & .044 & \\
\hline 1.3702 & .3576265 & .0553031 & \\
\hline 6054 & .3871215 & .0700506 & \\
\hline 75 & & .0589899 & \\
\hline $\begin{array}{l}826 \\
026\end{array}$ & $\begin{array}{l}\begin{array}{l}401011 \\
540480\end{array} \\
546\end{array}$ & $\begin{array}{l}.0700506 \\
.0405556\end{array}$ & \\
\hline & 540486 & & \\
\hline
\end{tabular}

$2.3024 \quad .0995455 \quad .0442425$

?.8307 $-0516162,0516167$

-3- P I pULARILEUI ELASIIL SLAIIERING SaSEL HUL LUNYENIUUN

B PEACET ALRROR FORHARD POINTS INIERPDLATIONS FROM CLOER DATA FOR OSDT ONLY 2.374 GEVIC, PLAB

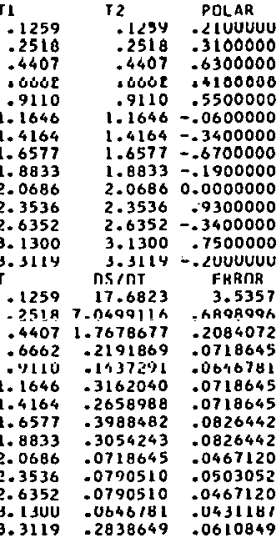

C $k$ - $P$ ELASTIC

C F-

C TIRST DIII HAS SCANMIMG RIAS SIGTOT $30.21-1$. SIGCL $5.7,-.26$

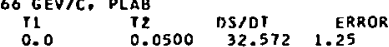

$\begin{array}{llll}11 & 12 & \text { DS7DT } & \text { ERROR } \\ 0.0 & 0.0500 & 32.572 & 1.25 \\ 0.0500 & 0.1000 & 22.2000 & 0.7021 \\ 0.1000 & 0.1500 & 15.4026 & 0.968\end{array}$

$0.1000 \quad 0.1500 \quad 15.4826 \quad 0.5863$

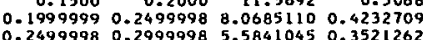

$\begin{array}{llll}0.2499998 & 0.2999998 & 5.5841045 & 0.3521262 \\ 0.2999998 & 0.3499991 & 3.8646812 & 0.2929398\end{array}$

006950.2541987

$0.3999997 \quad 0.4499996 \quad 1.98337170 .209857$

0.49999960 .54999950 .95731310 .1457970

-3. K- P ELASTIC

C $P$ - $P$. ELASTIC 19.441 ITALY BUBBLE
C

3. 0 GEV/C PLAB

ERROR 

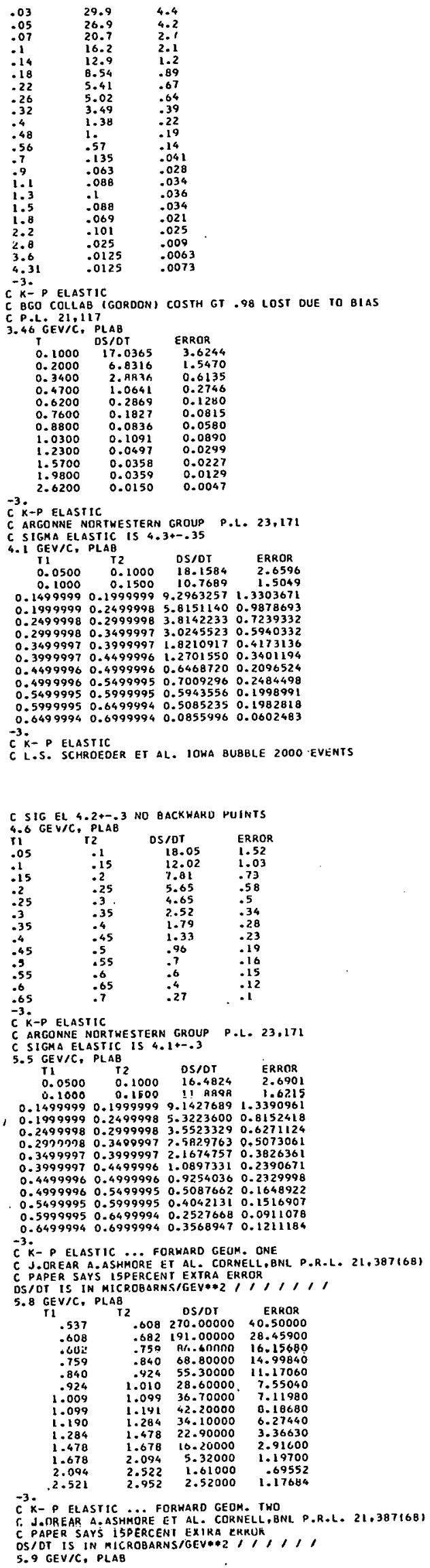

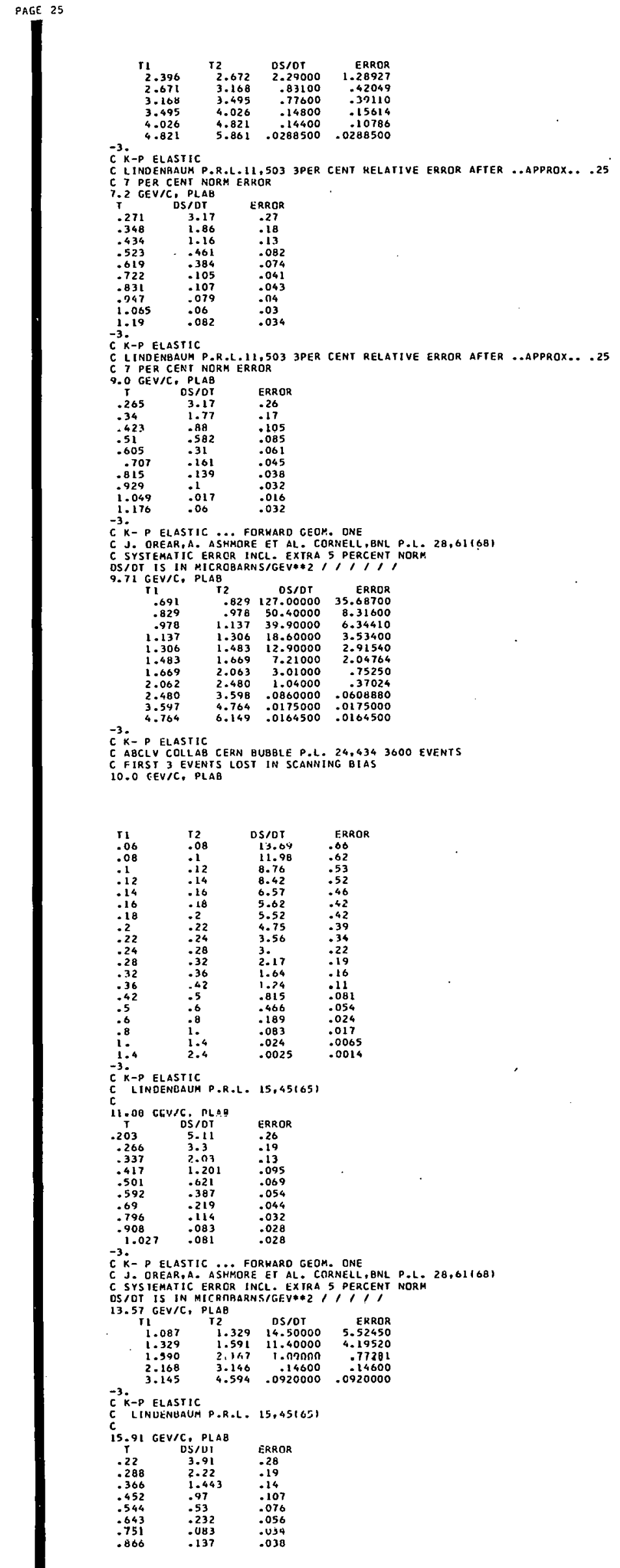




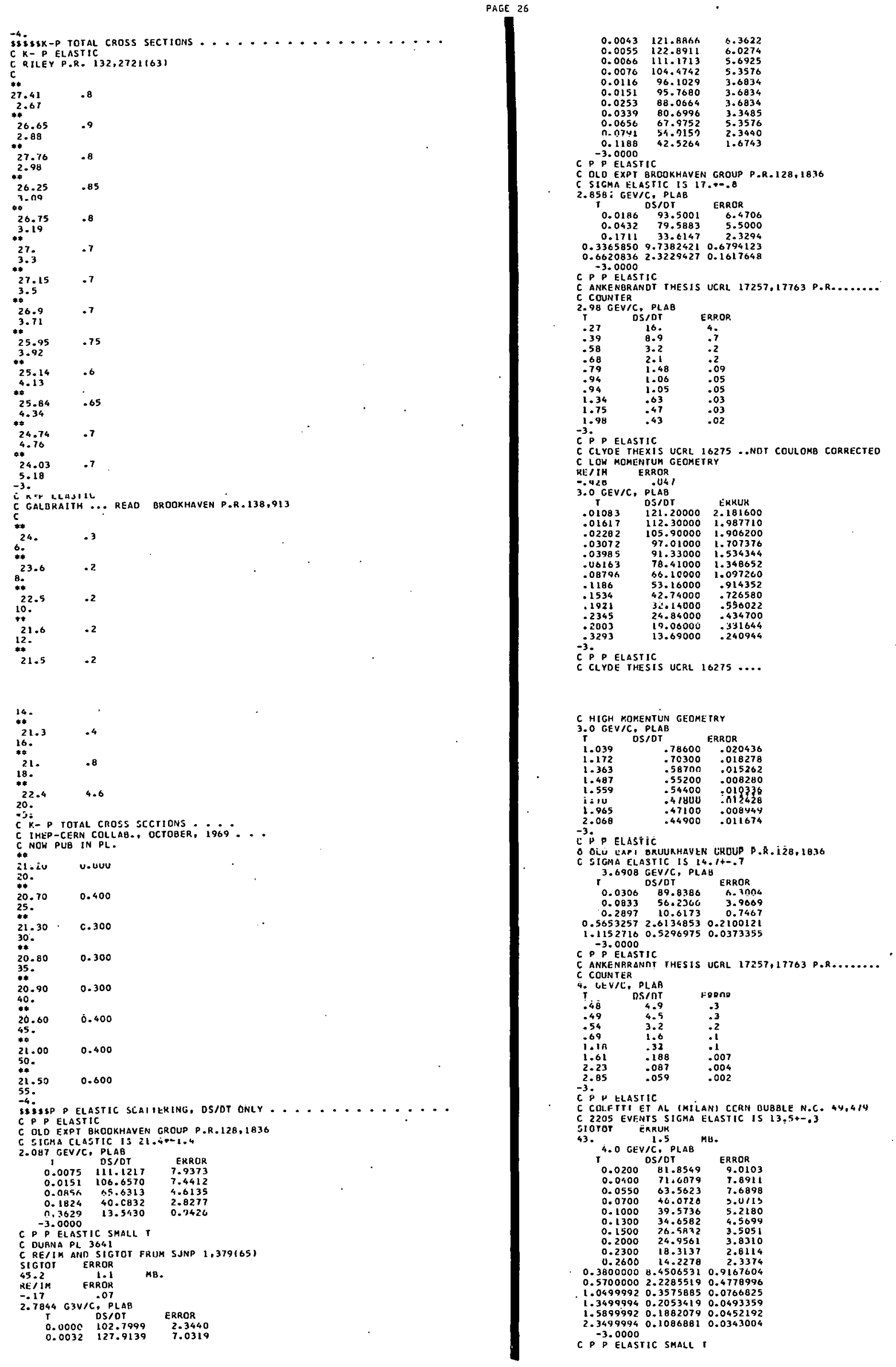




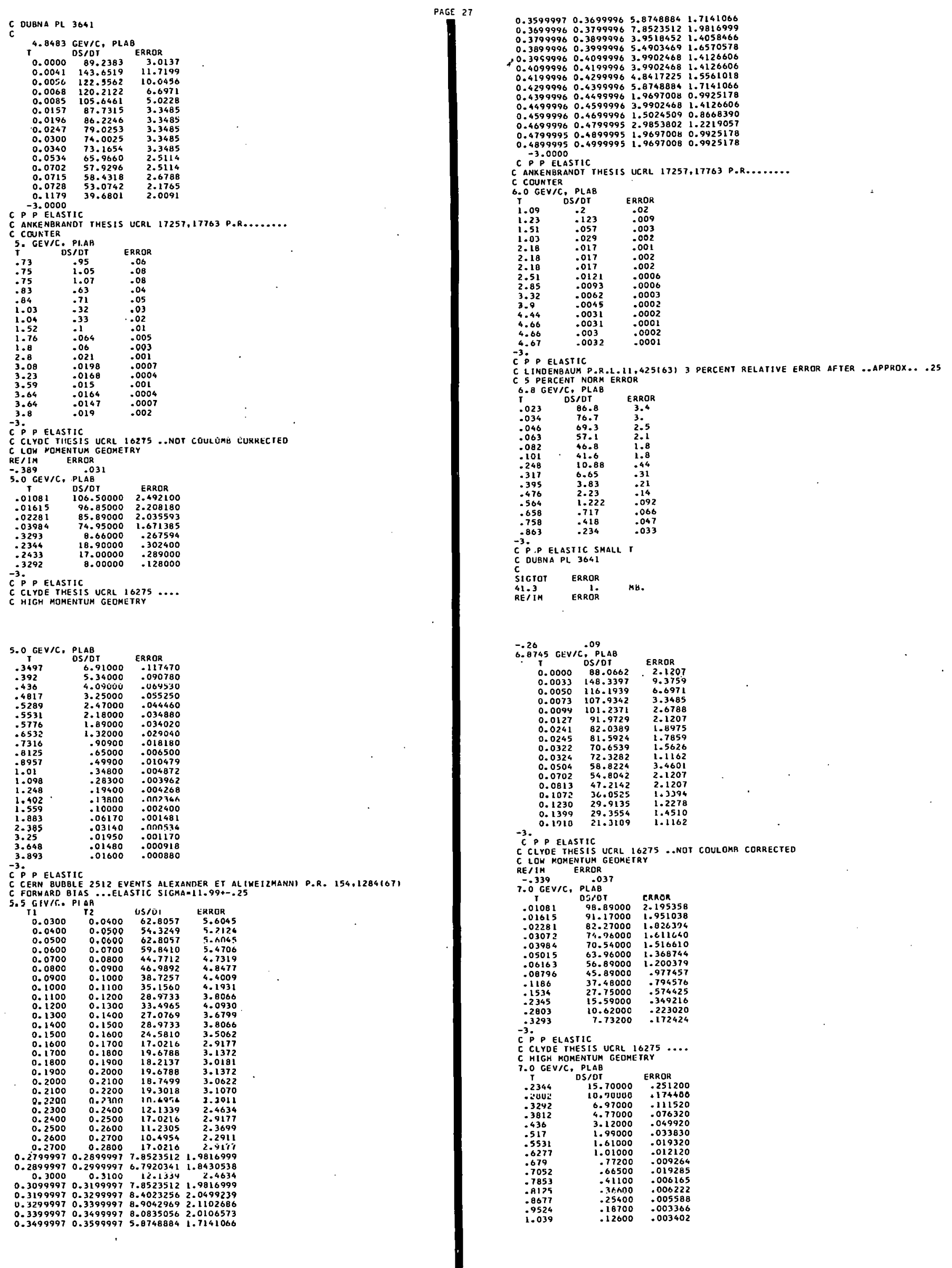




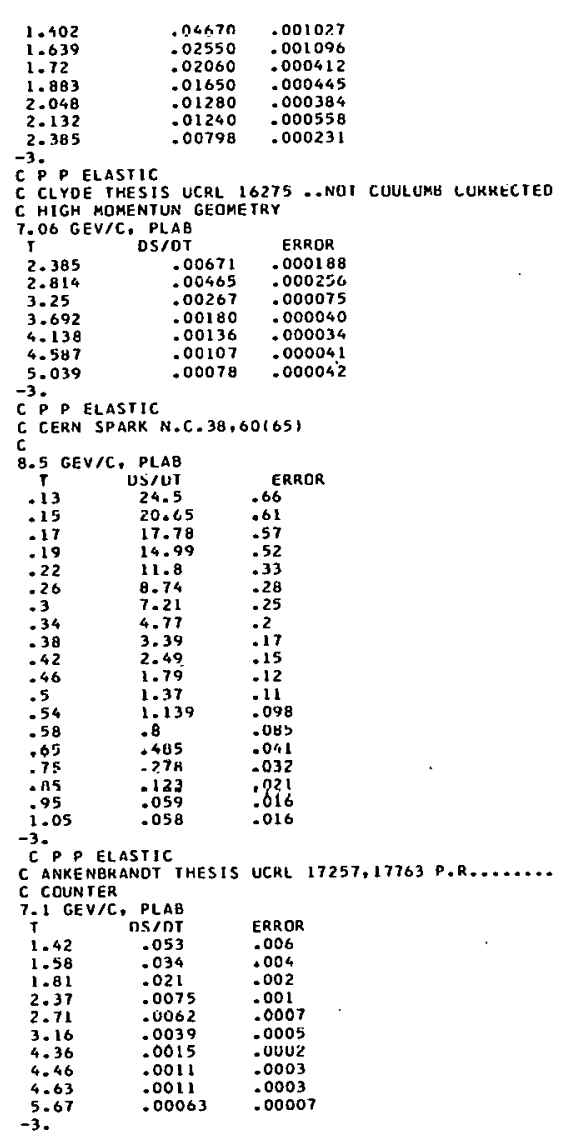

C P P ELASTIC

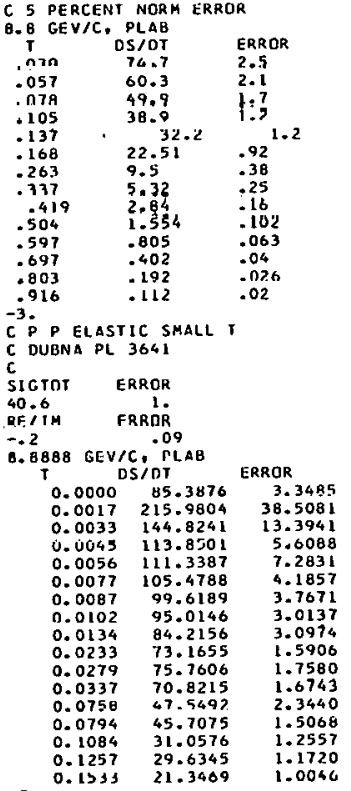

$c^{-3} \dot{p} \rho$ ELASTIL

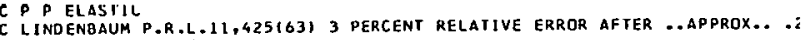
C5 PERCENT NORM ERRUA

\begin{tabular}{|c|c|}
\hline 10.8 & 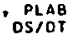 \\
\hline .058 & 59.4 \\
\hline & $\begin{array}{r}44.8 \\
32.7\end{array}$ \\
\hline $\begin{array}{r}-157 \\
: 204\end{array}$ & 23.73 \\
\hline .24 & 10.26 \\
\hline $\begin{array}{l}343 \\
343\end{array}$ & 4.53 \\
\hline & $\begin{array}{l}2.38 \\
1.148\end{array}$ \\
\hline
\end{tabular}

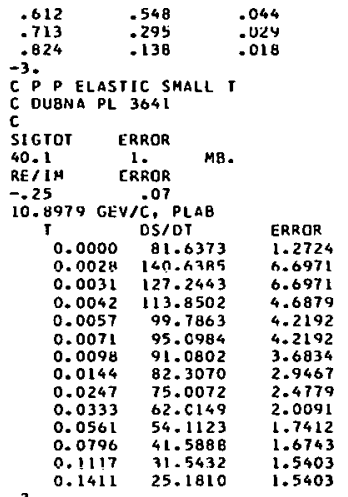

$c^{-3} \dot{p}$ P ELASTIC

c LINDENBaUh P.R.L. 15,451651

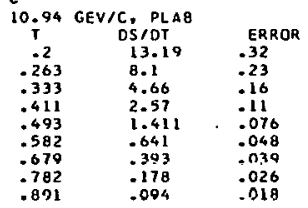

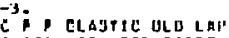

C CERN COUNTER DIDDENS.... WETHERALL P.R.L.9,1081621

12.1 GEV/C. PLAS

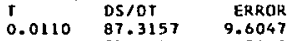

$\begin{array}{lll}0.0520 & 57.4130 & 4.5930 \\ 0.1210 & 26.9124 & 1.3930\end{array}$

$0.2140 \quad 12.7385 \quad 0.0369$

C. P P ELASTIC

C CERN SPARK N.C.. 38.60165

12.4 GEV/C, PLAB

$\begin{array}{ccc}r & \text { DS/DT } & \text { ERROR } \\ : 13 & 23.43 & .77 \\ : 15 & 18.4 & .65 \\ .17 & 16.22 & .61\end{array}$

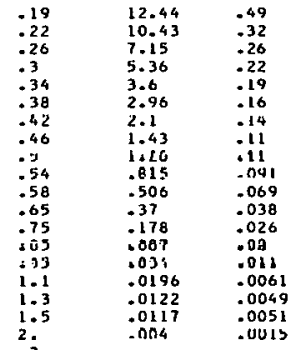

CP.P ELASTIC

作 C 5 PERCENT NORM ERROR

12.8 GEV/C, PLAB

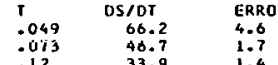

$\begin{array}{lll}.12 & 33.9 & 1.4 \\ .160 & 22.9 & 1.1 \\ .224 & 13.93 & .78 \\ .283 & 7.84 & : 54 \\ .290 & 0.84 & : .28\end{array}$

7.84

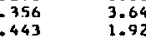

$\begin{array}{lll}.443 & 1.92 & 011 \\ .534 & .92 & .062 \\ .634 & .42 & .036\end{array}$

$\begin{array}{lll}. .741 & .42 & .029 \\ .856 & .036 \\ .091 & .025\end{array}$

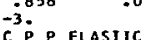

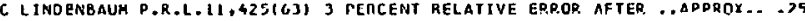
C5 PEREENT NORM ERROR

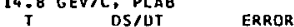

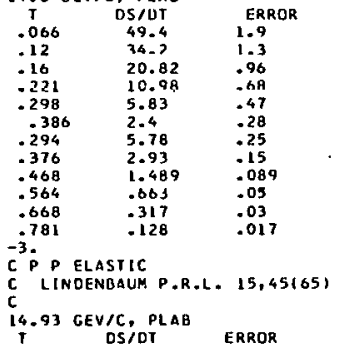




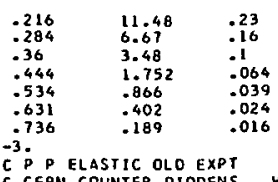

C CERN COUNTER DIDDENS... METHERALL P.R.L.9,100162) C EXIRA SYSTEMATIC ERROR IOPERCENT

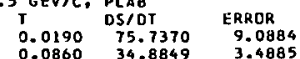

$0.1950 \quad 12.3933 \quad 0.7436$

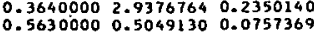

-3.

C P P ELASTIC C 5 PERCEHT NORH ERROR

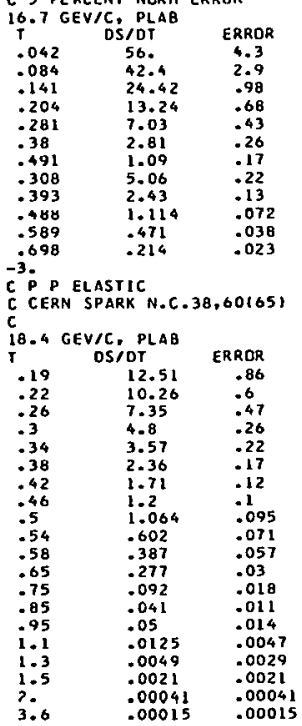

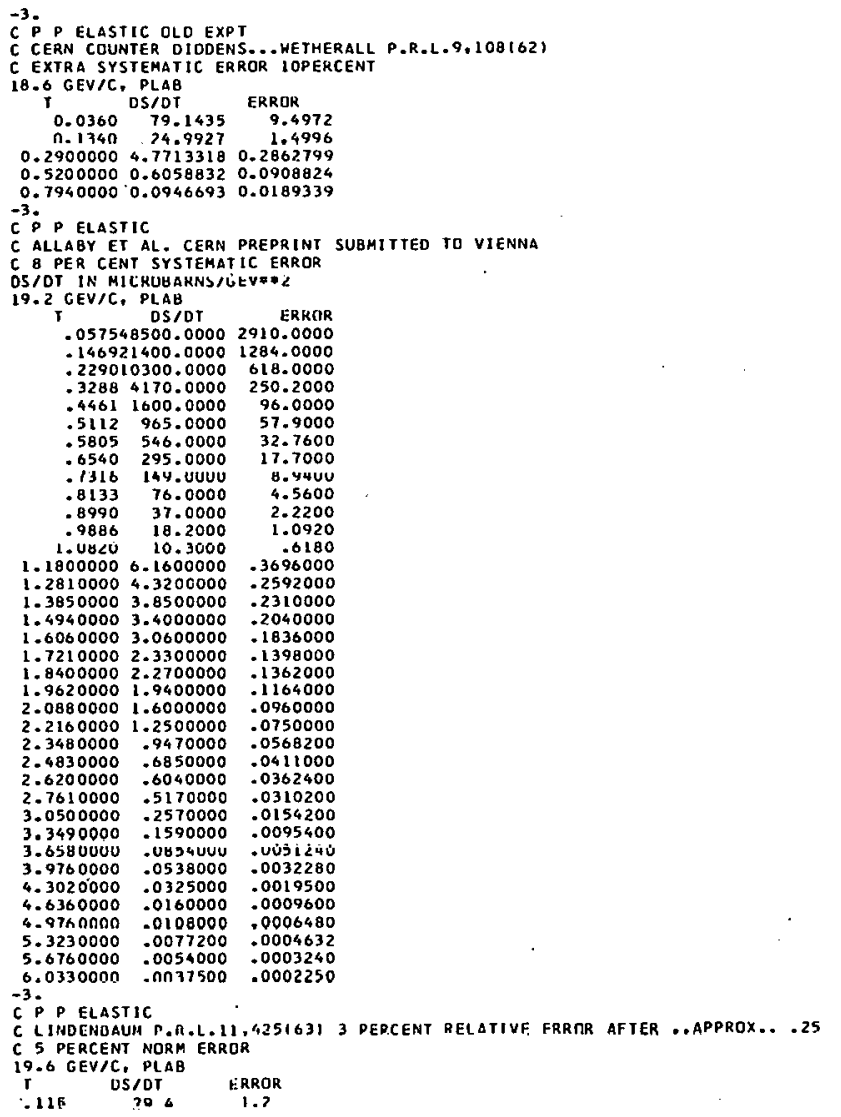

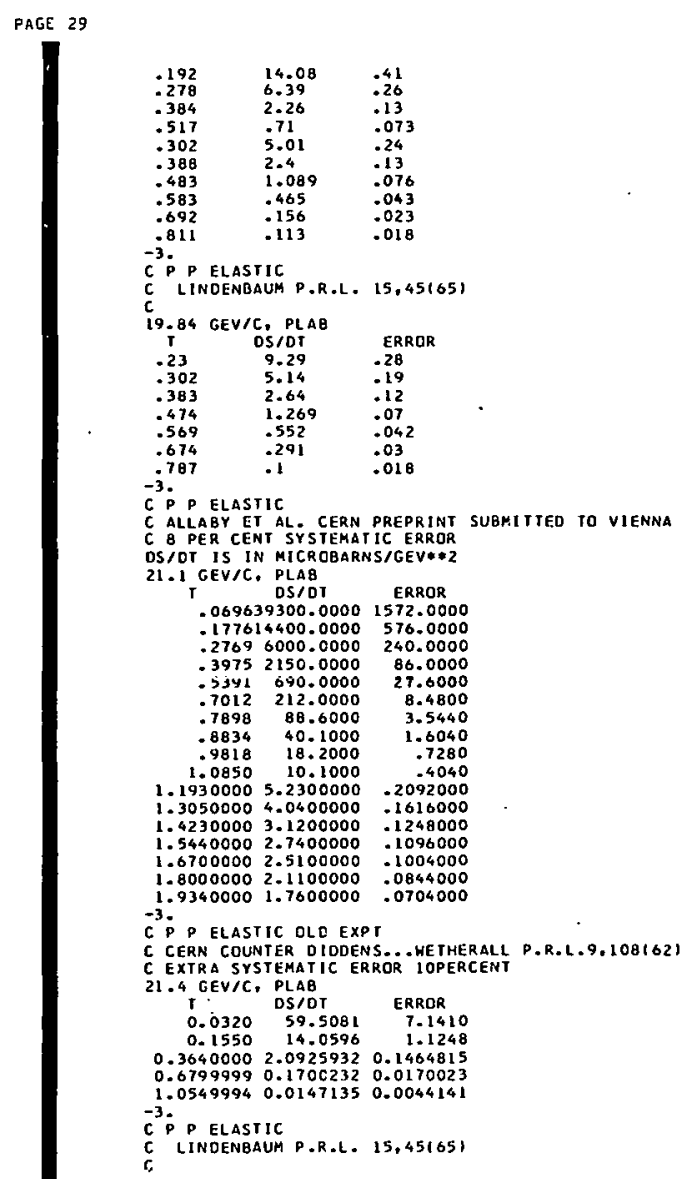

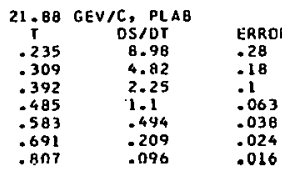

çP p ELASTIC

C LINDENBAUM P.R.L. 15,451651

24,63 GEV C DS PLAB

$\begin{array}{lll}T & \text { DS/OT } & \text { ERROR } \\ .254 & 7.56 & .34 \\ .334 & 3.14 & .21 \\ .424 & 1.712 & .122 \\ .525 & .824 & .074 \\ .632 & .203 & .036 \\ .748 & .11 & .028\end{array}$

$c^{-3} \dot{p}$ P ELASTIC OLD EXP

C P P ELASTIC OLD EXP T

C CERN COUNTER OIDDENS... HETHERALL P.

26.2 GEV/C. PLAG

0.0640 DS/OV B.U118 ERROR

$\begin{array}{llll}0.5960000 & 0.3285135 & 0.0328513 \\ 1.0419998 & 0.0198698 & 0.0039739\end{array}$

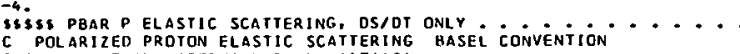

C C.DAUM ET AL. ICERN) N.P. 1.73 GEV/C. PLAB

T1. T2 POLAR ERROR

$\begin{array}{llll}.0966181 & .0966181 & .2700000 & .0800000\end{array}$

$\begin{array}{rrrr}.1449272 & .1449272 & .3300000 & .0400000 \\ .1739126 & .1739126 & .2800000 & .0400000\end{array}$

$\begin{array}{rrrr}.1739126 & .1739126 & .2800000 & .0400000 \\ .2415453 & .2415453 & .3400000 & .0700000\end{array}$

$\begin{array}{rrrrr}.3864724 & .3864724 & .6600000 & \mathbf{6 2 0 0 0 0 0} \\ .4251197 & \mathbf{4 2 5 1 1 9 7} & \mathbf{5 5 0 0 0 0 0} & \mathbf{1 1 7 0 0 0 0 0}\end{array}$

$.4830905 \quad .4836905 \quad .2900000 \quad .1700000$

$.5893704 \quad .5893704 \quad-1100000 \quad: 1400000$

$\begin{array}{rlll}.7342976 & .7342976 & .1900000 & .1600000 \\ .8309157 & .8309157 & .6200000 & : 1700000\end{array}$

$\begin{array}{llll}.8985484 & .8985484 & .3300000 & .2100000\end{array}$

$\begin{array}{rrrr}.9661811 & .9661811 & \$ 4500000 & \mathbf{2} 1900000\end{array}$

$1.1110821 .1111082 \quad .1800000 \quad .2100000$

$.22704091 .7270499 \quad .5700000 \quad .5000000$

$.25603541 .2560354-.2500000 \quad .4000000$

$1.3719771 \quad 1.3719771-.3200000 \quad .6500000$

$.4009251 .4009625 \quad-.3300000 \quad-4100000$

$\begin{array}{llll}.5169043 & 1.5169043 & 1.1600000 & 1.1300000 \\ .5458897 & 1.5458897 & -.8200000 & .6100000\end{array}$

$1.6714932 \quad 1.6714932 \quad .4900000 \quad .7100000$ 


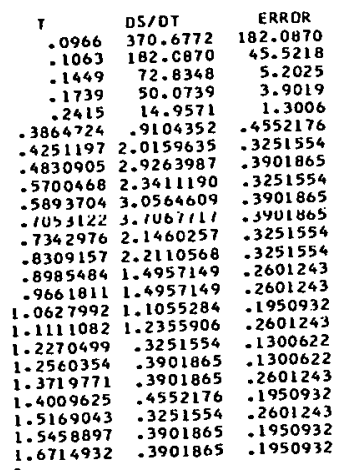

-3. polarized proton elastic scattering hasel convention

C C.DAUM ET AL. ICERNi N.P. 86,617160 )

C MARGINAL STATISTICS DUBIOUS NORM PR

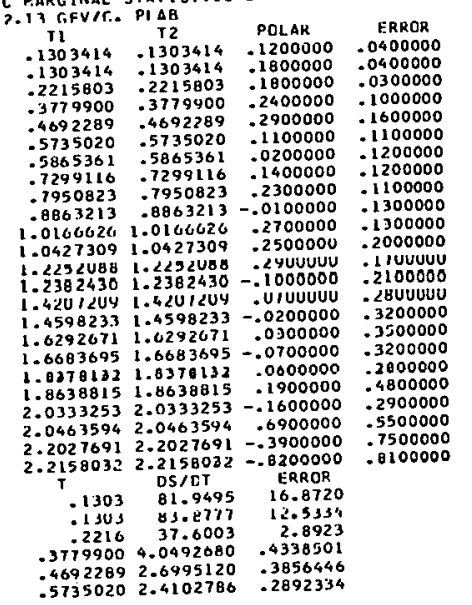

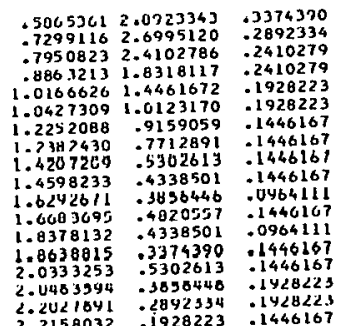

$c^{-3}$ polarized proton elastic scattering basel convention

C C.DAUM ET AL. TCERMI M.r. B6, G1716B] C MARG INAL STATISTIC
2.37 GEV/C. PLAB

\begin{tabular}{|c|c|c|c|c|}
\hline 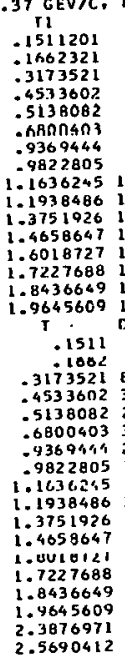 & 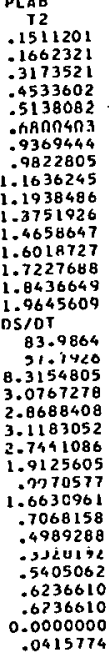 & 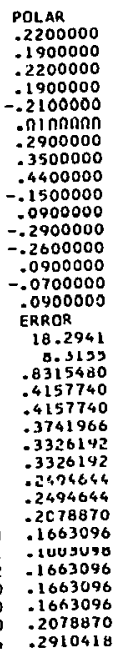 & $\begin{array}{l}\text { ERROR } \\
.0400000 \\
.0400000 \\
.0900000 \\
.1300000 \\
.1400000 \\
: 11000100 \\
-1100000 \\
: 1800000 \\
.2600000 \\
.1500000 \\
: 3000000 \\
.3600000 \\
.5100000 \\
.3200000 \\
.2600000 \\
.3100000\end{array}$ & . \\
\hline & & & & SEL CONVEN \\
\hline
\end{tabular}

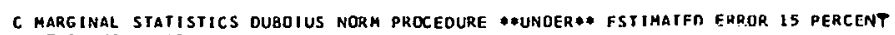
2.97 GEV/C, PLAB TI POLAR ERROR

$\begin{array}{rrrr}.1633565 & .1633565 & .0900000 & .0300000\end{array}$

$\begin{array}{llll}-3471326 & .3471326 & : 1700000 & .0500000 \\ -5309087 & .5309087 & : 1300000 & : 1600000\end{array}$

$.6330066 \quad .6330068-.0900000 \quad .1100000$

$.7759435 \quad .7759435 \quad .0300000 \quad-1500000$

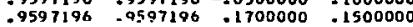

$\begin{array}{llllll}1.1843348 & 1.1843348 & .1300000 & -2100000\end{array}$

$1.3476914 \quad 1.3476914 \quad-1400000 \quad-1700000$

1.44976921 .4497892 .05000000 .2200000

$\begin{array}{llll}1.6948240 & 1.6948240 & -.5200000 & \mathbf{3} 300000 \\ 1.7152436 & 1.7152436 & -.1200000 & \mathbf{2 3 0 0 0 0 0}\end{array}$

$2.02153712 .0215371=-4200000 \quad+3800000$

$2.0623762 \quad 2.0623762-.0900000 \quad-2700000$

$\begin{array}{rrrr}2.3482501 & 2.3482501 & .4400000 & .6600000 \\ 2.6953827 & 2.6953827-2.2300000 & 2.6600000\end{array}$

2.6953827
2.7362219
2.7362219

$3.02209583 .0220958-.71000001 .2300000$

\begin{tabular}{l}
3.02209583 .0220958 \\
\hline
\end{tabular}

$\begin{array}{llll}3.2875502 & 3.2875502 & \mathbf{4} 2000000 & 1.3100000 \\ 3.3488089 & 3.3488089 & : 2900000 & 1.0600000\end{array}$

$\begin{array}{rrrr}3.6346828 & 3.6346828 & 1.2100000 & 3.2400000 \\ 3.0592980 & 3.8592980 & .7600000 & 2.9300000\end{array}$

T 15960 OS/OT ERROR

$\begin{array}{rrr}.1634 & 75.6952 & 16.0006 \\ .3471 & 19.3853 & 1.0462\end{array}$

$\begin{array}{rrr}.5309087 & .5231719 & .4307854\end{array}$

$.63300663 .0462685 \quad .3692447$

$.7994300 .9539574 \quad-338443$

$.95971962 .6462535 \quad .3692447$

$1.18433401 .0461932 \quad .1846223$

$\begin{array}{llll}1.3476914 & 1.1692748 & .1846223 \\ 1.4497892 & 1.1077340 & .2153927\end{array}$

$\begin{array}{rrr}1.4497892 & 1.1077340 & .2153927 \\ 1.6948240 & .5230966 & .1538519\end{array}$

$1.7152436 \quad .8923413 .1846223$

$\begin{array}{lll}2.0215371 & .4615558 & .1538510 \\ 2.0623762 & .5046374 & .1230816\end{array}$

$2.0623762 \quad .5046374 \quad .1230816$

-6957A27 -1320816 .1230010

$2.7742210 \quad 1064222 \quad-6598919$

$\begin{array}{llll}3.0220958 & .2153927 & .0972112 \\ 3.2675502 & .2153927 & .2401631\end{array}$

3.3488089 .1538519 .1230816

$\begin{array}{lll}9.0340024 & .0423112 & .1538519 \\ 3.0592980 & .1230416 & .2769335\end{array}$

$c^{-3}$ P pa

C BROOKHAVEN BUBBLE DOHINGO ET AL (BOULDER GRRIP) P.L. 24, G42 C SIGMA ELASIIC

TL 120180 OS/OT TRRTR

$\begin{array}{llll}0.0360 & 0.0360 & 224.2633 & 8.3706 \\ 0.0540 & 1 A 1.3638 & 7.3213\end{array}$

$0.0540 \quad 0.0721 \quad 133.2326 \quad 5.9292$

$\begin{array}{rrrr}0.0721 & 0.0901 & 115.0963 & 5.5804 \\ 0.0901 & 0.1081 & 89.6355 & 4.8829 \\ 0.1081 & 0.1261 & 62.4310 & 3.8365\end{array}$

$0.1261 \quad 0.1441 \quad 56.5018 \quad 3.8365$

$\begin{array}{llll}0.1441 & 0.1621 & 47.4336 & 3.4878 \\ 0.1621 & 0.1802 & 34.5638 & 2.7902 \\ 0.1802 & 0.2162 & 23.5773 & 1.6392\end{array}$

$0.2162 \quad 0.2522 \quad 12.8001 \quad 1.1858$

$0.2522116 \quad 0.2882414 \quad 7.25455090 .9068189$

0.32427120 .360301020 .2753580 .4882871

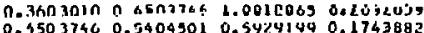

$\begin{array}{lllll}0 . \$ 503746 & 0.5404501 & 0.5424149 & 0.1743882 \\ 0.5404501 & 0.6305246 & 0.9416965 & 0.2092659\end{array}$

$\begin{array}{llll}0.5404501 & 0.6305246 & 0.9416965 & 0.2092659 \\ 0.6305246 & 0.7205992 & 1.3253508 & 0.2441435\end{array}$

$0.72069720 .010092,1.1100091$ U.1743892

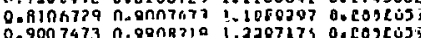

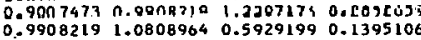

1.08089641 .044119450 .3941740 .0558042

1.441194511 .80149270 .24055560 .0453409

$2.16178942 .52208810 .0837063 \quad 0.0279021$

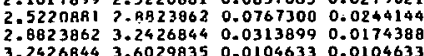

C. $P$ PBAR ELASTIC

C PEAR ELASTIC

SIGTOT ERROR

$70^{2}$
$3.0^{\circ}$ GEV/C. PLAB 1.5

of polarization

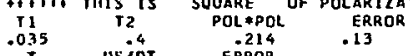

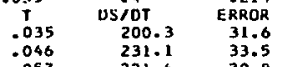

$\begin{array}{lll}.046 & 231.1 & 33.5 \\ .057 & 221.6 & 30.8 \\ .072 & 124.3 & 15.2 \\ .093 & 75 . & 11.2\end{array}$

$\begin{array}{lll}.093 & 75 . & 11.2 \\ .114 & 53.3 & 9.4 \\ .145 & 39.7 & 5.7\end{array}$

$\begin{array}{lll}.145 & 39.7 & 5.7 \\ .186 & 25.9 & 4.5 \\ .31 & 5.16 & 96\end{array}$

$\begin{array}{lll}.31 & 5.16 & .9 \\ .52 & 1.17 & .4 \\ .73 & .558 & .081 \\ .83 & .687 & .092\end{array}$

$\begin{array}{lll}.83 & .687 & .092 \\ 1.13 & .475 & .075 \\ 1.34 & .261 & .055 \\ 1.55 & .124 & .04\end{array}$

$\begin{array}{lll}1.55 & .124 & .04 \\ 1.75 & .167 & .044 \\ 1.96 & .106 & .035\end{array}$

$\begin{array}{lll}2.17 & .137 & 1161 \\ 2.37 & .0592 & .026\end{array}$

$\begin{array}{lll}2.58 & .0592 & .026 \\ 2.79 & .0235 & .026 \\ 3.31 & .0172 & .017\end{array}$

$\begin{array}{lll}3.31 & .0172 & .01 \\ 4.03 & .009 & .009\end{array}$

C. PRgar elastic

BROOKMAVEN A.G.5. YALE, BNL, CITY NY, COLLAB P.R. 137,1250 C SIGMA ELASTIC $21.94-1.1$ 

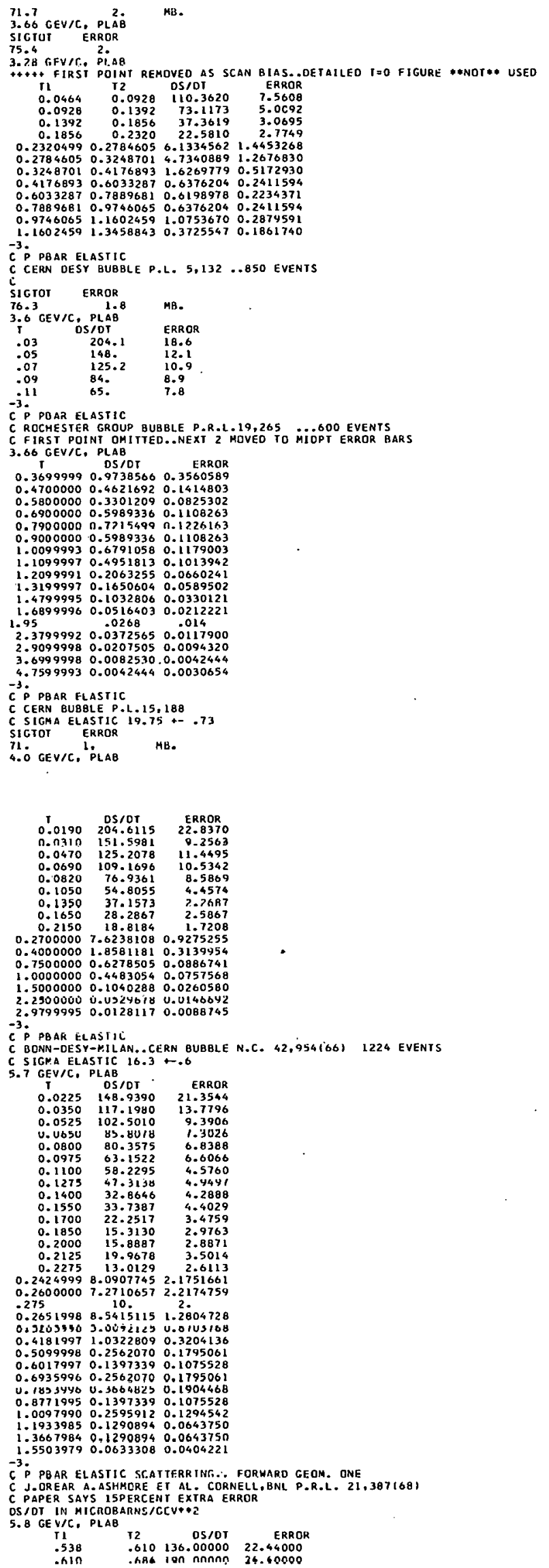

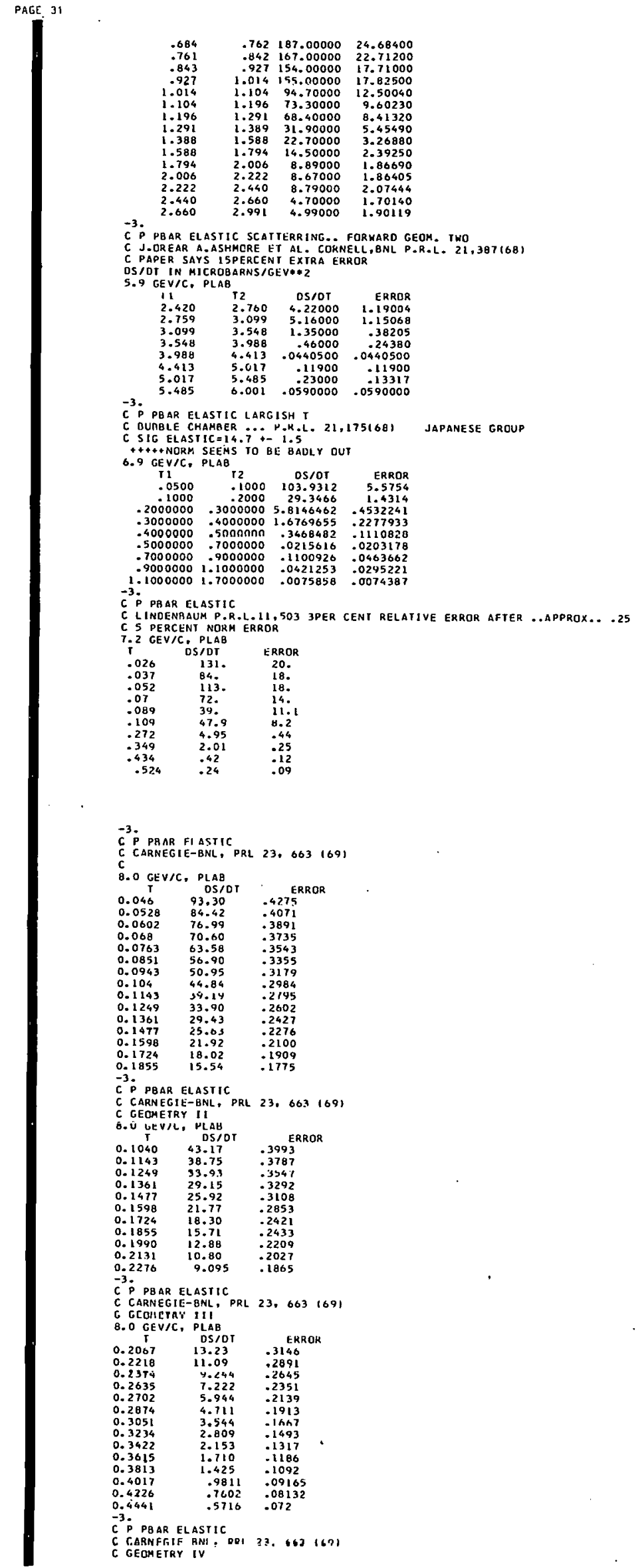



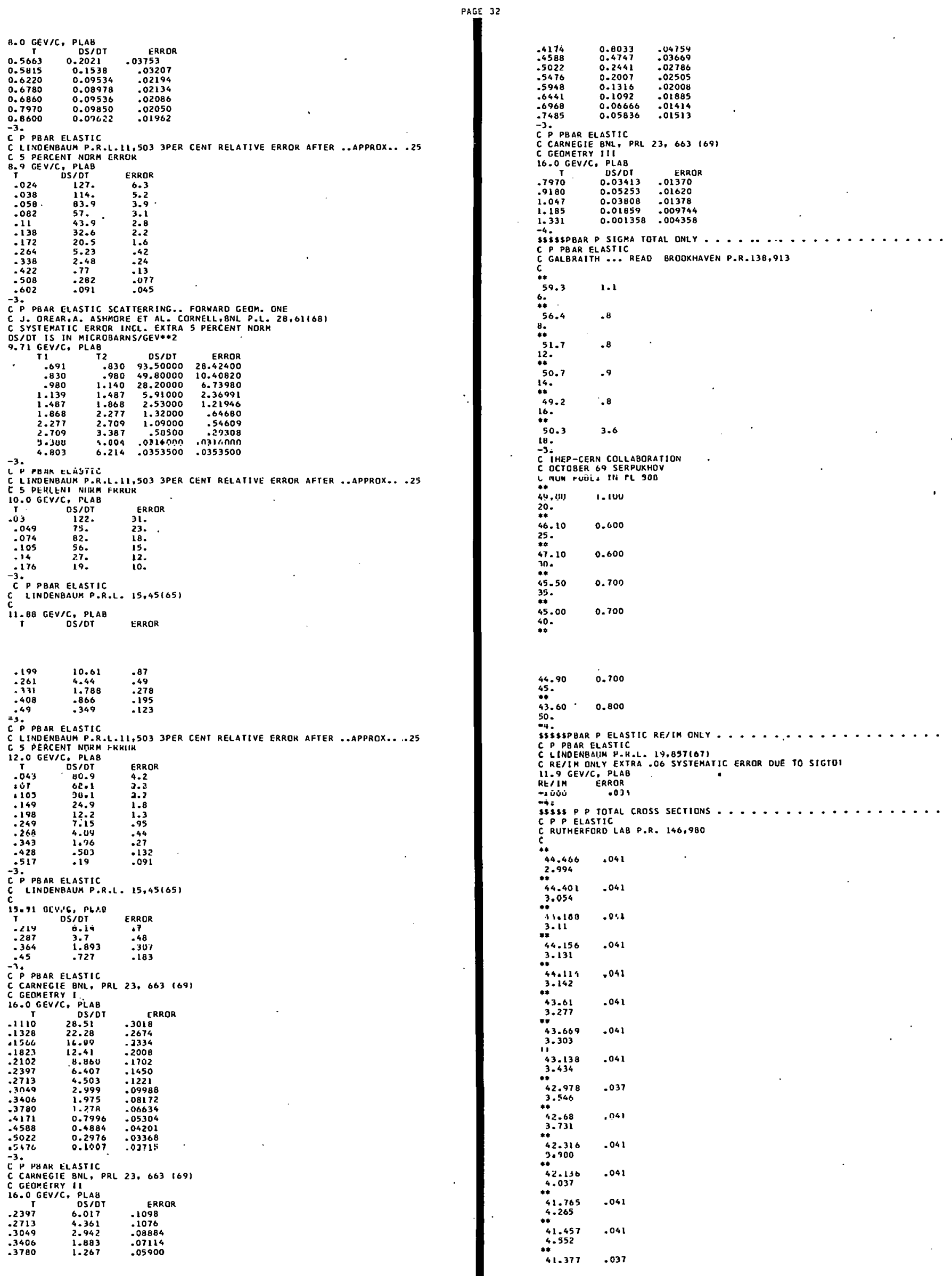


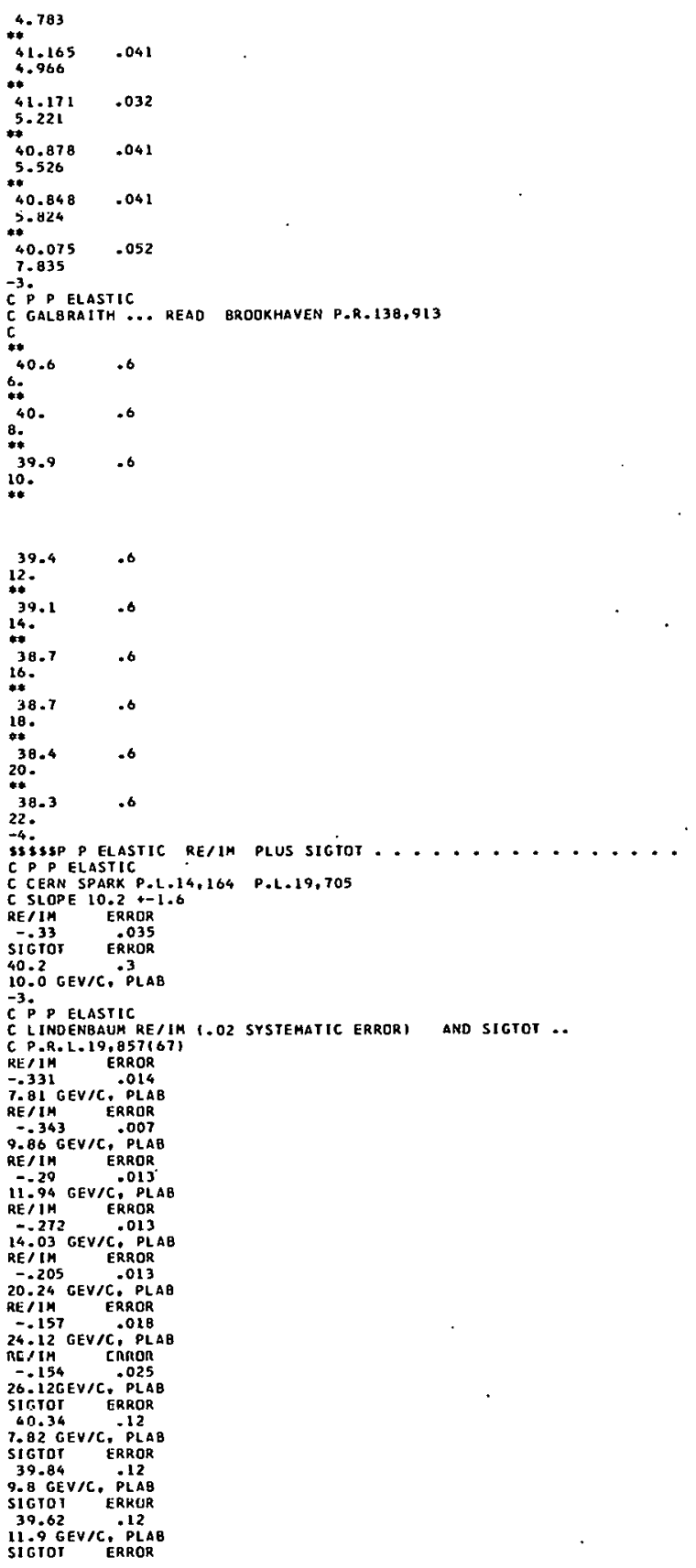

C BERKELEY GROUPICHAMBERLAIN ET ALI P.R. 148,1297166$)$ 3.6 GEV/C, PLAB

\begin{tabular}{|c|c|c|c|}
\hline${ }_{\text {T1 }}^{6}$ GEV $/ C$. & $\begin{array}{c}\text { PLAB } \\
\text { T2 }\end{array}$ & & \\
\hline-111 & -111 & .151 & .085 \\
\hline $\begin{array}{l}146 \\
.185\end{array}$ & $\begin{array}{l}146 \\
: 185\end{array}$ & $\begin{array}{r}188 \\
.237\end{array}$ & $\begin{array}{l}.07 \\
.015\end{array}$ \\
\hline $\begin{array}{r}-228 \\
-275 \\
0\end{array}$ & .228 & .245 & .015. \\
\hline & . .326 & .26 & .02 \\
\hline $\begin{array}{l}0 \\
05\end{array}$ & $\begin{array}{l}-38 \\
.405\end{array}$ & .221 & $\begin{array}{l}.022 \\
.019\end{array}$ \\
\hline .438 & .438 & .283 & .028 \\
\hline & $\begin{array}{r}-463 \\
-524\end{array}$ & $\begin{array}{r}-242 \\
.225\end{array}$ & $\begin{array}{r}.022 \\
.023\end{array}$ \\
\hline 88 & .508 & .190 & .027 \\
\hline 24 & $\begin{array}{l}-724 \\
-724\end{array}$ & .218 & .036 \\
\hline $\begin{array}{l}96 \\
69\end{array}$ & $\begin{array}{l}.796 \\
.869\end{array}$ & $\begin{array}{l}156 \\
.13\end{array}$ & $\begin{array}{l}.04 \\
.04 ?\end{array}$ \\
\hline $\begin{array}{l}943 \\
.02\end{array}$ & $\begin{array}{l}943 \\
i .02\end{array}$ & $\begin{array}{l}171 \\
.104\end{array}$ & .092 \\
\hline
\end{tabular}

$c^{-3} \dot{P}$ P ELASTIC POLARIZATION ONLY

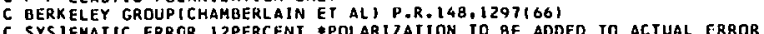
4. 34 GEVIC, PLAB 12 PERCENT OPOL ARIZATI

\begin{tabular}{|c|c|c|c|}
\hline & & & \\
\hline 11 & 12 & POLAR & ERROR \\
\hline 215 & .215 & .171 & .019 \\
\hline .257 & .257 & .203 & .021 \\
\hline & .304 & .203 & .024 \\
\hline .353 & .353 & .218 & .028 \\
\hline .405 & .405 & .207 & .113 \\
\hline .461 & .461 & .224 & .035 \\
\hline .519 & .519 & .131 & .046 \\
\hline .58 & .58 & .123 & .044 \\
\hline .643 & .643 & .083 & .054 \\
\hline 109 & .707 & .127 & nha \\
\hline & $\begin{array}{l}\text { IC PO } \\
\text { GRDUP } \\
\text { C ERR }\end{array}$ & $\begin{array}{l}\angle A I 10 N \\
\text { MQERLAI } \\
\text { ZPERCEN }\end{array}$ & \\
\hline $6.85 \mathrm{C}$ & & & \\
\hline II & 12 & POLAR & ERROR \\
\hline .138 & .138 & .144 & .025 \\
\hline & .176 & $01 \% 1$ & .016 \\
\hline .218 & .218 & .211 & .015 \\
\hline .264 & .264 & .193 & .017 \\
\hline .314 & .314 & .217 & \\
\hline .368 & .368 & .181 & .022 \\
\hline 35 & .425 & .194 & .026 \\
\hline
\end{tabular}


C P P ELASTIC POLARILATION 10 PERCENT NORY ERROR AGREES WITH 4.84

\begin{tabular}{|c|c|c|c|}
\hline \multicolumn{4}{|c|}{5.15 GEV/C, PLAB } \\
\hline Til & 12 & POLAR & ERROR \\
\hline .279 & .2279 & $\begin{array}{l}.219 \\
.216\end{array}$ & $\begin{array}{l}.013 \\
.012\end{array}$ \\
\hline .334 & .339 & .175 & .010 \\
\hline .406 & .406 & . 184 & .018 \\
\hline .487 & .487 & .172 & .016 \\
\hline .555 & .555 & .124 & .021 \\
\hline & .045 & .123 & .026 \\
\hline .737 & .737 & -151 & .031 \\
\hline .823 & .823 & .13 & .04 \\
\hline .916 & .916 & .135 & .052 \\
\hline 1.02 & 1.02 & .199 & .0585 \\
\hline 1.12 & 1.12 & .24 & .069 \\
\hline 1.23 & 1.23 & .26 & .082 \\
\hline 1.35 & 1.35 & .169 & .1 \\
\hline 1.45 & 1.45 & .25 & \\
\hline 1.57 & 1.57 & .185 & .133 \\
\hline 1.74 & 1.74 & .33 & .13 \\
\hline $\begin{array}{ll}C & p \\
C & B E \\
c & S Y \\
S & \end{array}$ & & & \\
\hline $\begin{array}{ll}3.9 \\
T 1\end{array}$ & 12 & POLAR & ERROR \\
\hline .112 & .112 & .089 & .1 \\
\hline .147 & .147 & .152 & .024 \\
\hline .186 & .186 & .166 & .019 \\
\hline .229 & .229 & $\begin{array}{r}153 \\
.178\end{array}$ & .019 \\
\hline .329 & .329 & .185 & .027 \\
\hline .384 & .384 & .136 & .03 \\
\hline .398 & .398 & .226 & .054 \\
\hline & .444 & .136 & .034 \\
\hline .459 & .459 & .201 & .059 \\
\hline .523 & .523 & .149 & .055 \\
\hline .592 & .501 & . Ans & - U6? \\
\hline .062 & .662 & .231 & .007 \\
\hline .814 & .814 & & .121 \\
\hline .914 & .914 & .041 & .138 \\
\hline 0 & Ir. & & \\
\hline $\begin{array}{l}\text { C CER } \\
\text { C Fiv }\end{array}$ & in & .2 & 7671 \\
\hline C. Fiv & $\begin{array}{l}\text { ENT } \\
\text { PLAB }\end{array}$ & & \\
\hline 11 & 12 & POLAR & ERRD \\
\hline .109 & .109 & & \\
\hline .136 & .136 & .041 & .034 \\
\hline .166 & 166 & .122 & .025 \\
\hline 198 & 198 & . 138 & .016 \\
\hline .233 & .233 & .101 & .014 \\
\hline & .271 & .117 & $n 14$ \\
\hline 2 & .312 & . 0955 & .015 \\
\hline & .355 & .141 & .017 \\
\hline & & .117 & .018 \\
\hline 448 & .448 & .083 & .019 \\
\hline $\begin{array}{r}.499 \\
.551\end{array}$ & $\begin{array}{r}-499 \\
.551\end{array}$ & .063 & .029 \\
\hline
\end{tabular}

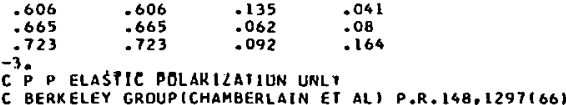

C BERKELEY GROUP (CHAMBERLAIN ET AL) P.R. 148, $1297(60)$ 7.0 GEV/C. PLAB

\begin{tabular}{|c|c|c|c|}
\hline \\
\hline $\begin{array}{l}11 \\
.152\end{array}$ & & POL AR & ERROR \\
\hline $\begin{array}{l}152 \\
.195\end{array}$ & $\begin{array}{r}152 \\
.195\end{array}$ & 0.112 & $\begin{array}{r}.049 \\
.031\end{array}$ \\
\hline $\begin{array}{l}-242 \\
0445\end{array}$ & .272 & 198 & .829 \\
\hline $\begin{array}{l}.245 \\
.352\end{array}$ & $\begin{array}{r}-293 \\
.352\end{array}$ & .262 & (037 \\
\hline - 513 & .41. & 110 & 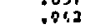 \\
\hline & .461 & .169 & .027 \\
\hline .48 & -4 & -157 & . U93 \\
\hline & & 0117 & \\
\hline & .39 & $\begin{array}{l}.077 \\
.085\end{array}$ & .064 \\
\hline & $\begin{array}{l}.0002 \\
.679\end{array}$ & .083 & .053 \\
\hline $\begin{array}{l}.759 \\
.759\end{array}$ & & .002 & .074 \\
\hline \multirow{4}{*}{\multicolumn{4}{|c|}{$\begin{array}{l}\text { C P P ELASTIC POLARIZATION } \\
\text { C KANAVEIS EI AL JINR } \\
\text { C. IFIP IB.874(64) } \\
9,3 \text { GEV/C, PLAB }\end{array}$}} \\
\hline & & & \\
\hline & & & \\
\hline & & & \\
\hline $\begin{array}{r}-11 \\
-.28\end{array}$ & $=.28$ & POLAR 02 & $\begin{array}{l}\text { ERRQR } \\
.06\end{array}$ \\
\hline \multirow{4}{*}{\multicolumn{4}{|c|}{$\begin{array}{l}\text { C P P ELASTIC } \\
\text { C CERN POLARIZATION P.L. } 24,771671 \\
\text { C FIVE PERCENT RELATIVE ERROR } \\
\text { I0.0 GEV/C. PLAB }\end{array}$}} \\
\hline & & & \\
\hline & & & \\
\hline & & & \\
\hline 102 & .102 & .074 & $\begin{array}{l}\text { ERROR } \\
.072\end{array}$ \\
\hline & .129 & .059 & .03 \\
\hline .159 & .159 & .058 & .023 \\
\hline & 192 & .097 & \\
\hline .22 & .2 & .093 & .013 \\
\hline & .267 & .071 & .013 \\
\hline & .309 & .082 & .014 \\
\hline 354 & .354 & .093 & .017 \\
\hline & . 401 & .081 & .018 \\
\hline & .452 & .067 & .019 \\
\hline 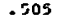 & .505 & .074 & .023 \\
\hline & .561 & .067 & .028 \\
\hline & & .077 & .037 \\
\hline & & .117 & .046 \\
\hline & .745 & .08 & .045 \\
\hline \multirow{4}{*}{\multicolumn{4}{|c|}{$\begin{array}{l}\text { C P P ELASIIC } \\
\text { C CERN POLARIZAIION P. }-24,24,771671 \\
\text { C FIVE PERCENT RELATIVE ERROR }\end{array}$}} \\
\hline & & & \\
\hline & & & \\
\hline & & & \\
\hline & & POLAR & RRO \\
\hline 3 & 103 & .034 & \\
\hline & .131 & .032 & .0 \\
\hline & .163 & .03 & .01 \\
\hline & & .042 & .013 \\
\hline & .2 & & \\
\hline 779 & .279 & .064 & .013 \\
\hline
\end{tabular}

$\begin{array}{llll}.324 & .324 & .041 & .014 \\ .373 & .373 & .055 & .017 \\ .424 & .024 & .031 & .019 \\ .579 & .479 & .046 & .019 \\ .0237 & .537 & .073 & .023 \\ .029 & .629 & .006 & .023 \\ .762 & .762 & .002 & .026\end{array}$

$C^{-3} P$ P ELASTIC

C R. T. EELL ET AL. CERN, ORSAY, PISA DRELIA RESUL ISIVIENNA

C EXTRA S PERCENT TARGEI POL AR SCALE ERROR

Ii i EEV T PLAB POLAR ERROR

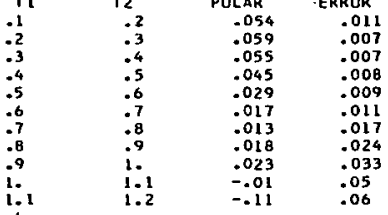

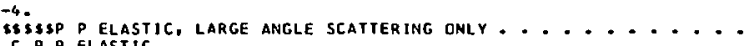

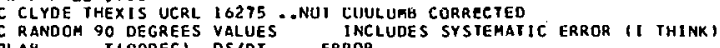

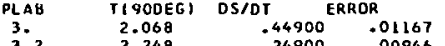

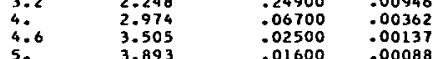

$\begin{array}{llll}5 . & 3.893 & : 001600 & .00008 \\ 6 . & 4.817 & .00335 & : 00018\end{array}$

$c^{-3} \dot{p}$ p elastic at 90 oegrees ... ENS.

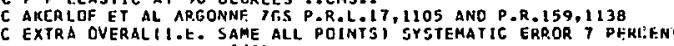

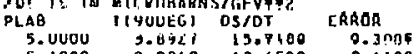
$\begin{array}{llll}5.0000 & 9.0921 & 13.7100 & 0.3004 \\ 5.1000 & 3.9849 & 12.4500 & 0.4108\end{array}$

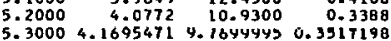
5.30004 .1695471 Y. 1844445 O. 3517198

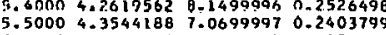
$5.60004 .4469242 \quad 6.31999970 .1959199$
$5.9000 \quad 4.7247076 \quad 3.63999940 .1273999$
$6.00004 .81738573 .1799994 \quad 0.0965799$
6.1000 4.9100952 $2.7999992 \quad 0.1035999$
6.4000 5.1804346 L 1 199997 0.0673400
0.60005 .3741493 L.25000nn 0.0587500
$6.80005 .55997470 .9000000 \quad 0.0423000$
$7.00005 .7459078 \quad 0.7060000 \quad 0.0289460$
$7.6000 \quad 6.3042173 \quad 0.3040000 \quad 0.0164160$

$7.8000 \quad 6.4904814 \quad 0.24499990 .0110250$

$8.0000 \quad 6.6768141 \quad 0.2040000 \quad 0.0091800$

8.10006 .76999660 .15699990 .0061230

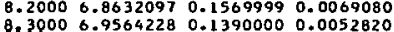

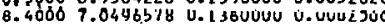

8.60007 .23615460 .11000000 .0050600

9.00007 .42271800 .08710000 .009

$.20007 .7959785 \quad 0.0628999$
0.0027047

$9.40007 .9826660 \quad 0.0532000 \quad 0.002819$

90008.16939450 .04530000 .0022197

0.0000 8.5423668 0.03440000 .001685

$0.2000 \quad 8.72980310 .0310000 \quad 0.0015264$

lo. 4000 8.

10.6000 9.8141

11.00009 .47744270 .01800000 .0010340

$1.40009 .85141370 .0129000 \quad 0.000696$

11.600010 .03843210 .01190000 .000618

11.

$\begin{array}{lll}2.000010 .9738188 & 0.0054500 & 0.000343\end{array}$

$.800011 .16094590 .0048800 \quad 0.000218$

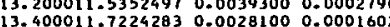

-3.0000

C P P ELASTIC

C OROOKHAVEN COUNTER EXPT.. OREAR ET AL P.R.152.1162

CS LARGE IS IN MICROBARNS/GEV**2

8.0 GEV/C. PLAH

8. TEV $/ C$ PLAB
OS/OT ERROR

$\begin{array}{lll}1.74 & 14 . & 1.4 \\ 2.23 & 3: 0 \mathrm{E} & .0\end{array}$

$\begin{array}{lll}1.5 & 4.99 & .59 \\ 2.72 & 3.47 & .58 \\ 3.5 & 2.27 & .31 \\ 3.5 & 1.54 & .26\end{array}$

C. P. E ELASTIC

C P ELASTIC COUNTER EYOT TRESR ET AL P.R ISTO 1162

C LARGE I

OS/OT IS IN MICROBARNS/GEV*\#2

12.0 GEYIC, PLAB DSTOT ERROR
T

$\begin{array}{rrr}T & \text { DSROT } & \text { ERROR } \\ 1.2 & 16.8 & 1.5 \\ 1.5 & 8.62 & .80 \\ 2.5 & 5.31 & .69 \\ 2.4 & 2.19 & .54 \\ 2.5 & 1.99 & .66\end{array}$

-3. P P ELASTIC AT LARGE ANGLE

(CERN) CERN 68-7 PAGE 580 P.L. 27,149168 ERROR $7 \ldots 12$ GEV OF SEVEN PERCENT

7.1 GEVIC. PLAB 


$$
\begin{array}{lll} 
& & \\
T & \text { DS/OT } & \text { ERROR } \\
2.9194 & 4401.2026 & 176.0481 \\
3.0977 & 3766.3103 & 150.6524 \\
3.3713 & 2733.2652 & 109.3306 \\
3.6516 & 2152.1773 & 86.0871 \\
3.9379 & 1665.7852 & 66.6314 \\
4.3277 & 1263.3281 & 50.5331 \\
4.8250 & 894.2297 & 35.7692 \\
5.8389 & 672.5554 & 26.9022
\end{array}
$$

c. $P$ P elastic at large angle

C ALLABY..... HE THERALL (CERNI CERN $68-7$ PAGE 580 C COMHON SCALE ERROR $7 \ldots 12$ GEV OF SEVEN PERCEN

P.L. 27,149168 )

Q.1 GEV/C, IN NANOBARNS/GEV*\#2

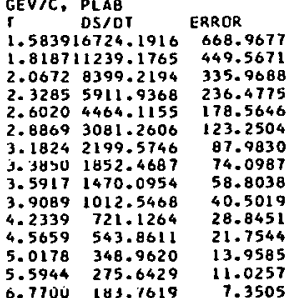

c); p Elastic at large angle

ALLABY..... HETHERALL ICERNI CERN 68-7 PAGE SBO P.L. 27,149168 C COMMON SCALE ERROR $7 . .12$ GEV. OF SEVEN PERCENT

9.2 GEV/C, PLAB

ERROR

$1.82397084 .3060 \quad 283.3722$

$2.09445391 .8097 \quad 215.672$

$2.38044142 .5862 \quad 165.7034$

2.99831950 .4005

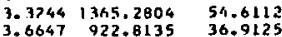

$\begin{array}{lll}3.6647 & 922.8135 & 36.9125 \\ 3.8980 & 681.0283 & 27.2411\end{array}$

3. 8980081.0283

$4.1360 \quad 496.4656$

0.5108

5.7782 $117.4270 \quad 4.697$

$\begin{array}{lll}6.4422 & 89.5217 & 3.5849 \\ 7.7960 & 84.7054 & 3.3882\end{array}$

$\vec{c} p$ p elastic at large angle

C ALLAQY... HETHERALL ICERNI CERN GQ-7 PAGE SBO
C COMMCN SCALE ERROR 7...12 GEV OF SEVEN PERCENT DS DOT IS IN NANOBARS GeVä

10.1 GEV/C. PLAB

2.02054343 .3150 ERROR

$\begin{array}{lll}2.0205 & 4343.3150 & 173.73326 \\ 2.2193 & 4059.5808 & 162.3832\end{array}$

\section{$\begin{array}{rrr}2.4239 & 3266.5803 & 130.6632 \\ 2.6370 & 2480.8549 & 99.2342\end{array}$ \\ 2.85752826 .0838 \\ 3.0850 1356.8312 \\ $\begin{array}{lll}3.5600 & 758.1484 & 38.325 \\ 318.7932 & 28.7517\end{array}$ \\ $\begin{array}{lll}3.8070 & 468.5251 & 18.7410\end{array}$ \\ $\begin{array}{lll}4.0598 & 349.9388 & 13.9976 \\ 4.3182 & 250.9956 & 10.0398\end{array}$ \\ $4.5818 \quad 185.5185 \quad 7.4207$

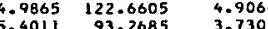 \\ $\begin{array}{lll}5.8247 & 67.9507 & 2.73180 \\ 5.4011 & 53.1820 & 2.127\end{array}$ \\ $7.1367 \quad 43.6514$}

$\vec{c}^{3}$ p p elastic at LARGe angL

C ALLABY......NE THERALL (CERN) CERN 68-7 PAGE 580 P.L. 27,149168) OS IOT IS IN NAMAORNSICEVE*

$$
\begin{aligned}
& \begin{array}{ll}
\text { GEV/C. PLAB } & \text { DS/DT } \\
\text { ERROR } \\
2.2392 \\
3367.7710 & 134.710
\end{array} \\
& 7710 \quad 134.710 \\
& \begin{array}{lll}
2.9224 & 1319.5360 & 52.7814
\end{array} \\
& 3.2918743 .1873 \quad 31.327 \\
& \begin{array}{rrr}
3.0183 & 431.2144 & 17.488 \\
4.0813 & 236.3348 & 9.4534
\end{array} \\
& 4.4991 \quad 146.3963 \quad 5.8559
\end{aligned}
$$

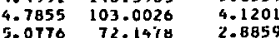

$$
\begin{aligned}
& 5.0770 \quad 72.1478 \quad 2.8859 \\
& \begin{array}{lll}
5.5261 & 50.6150 & 2.0240 \\
5.9856 & 37.5510 & 1.5020
\end{array} \\
& \begin{array}{lll}
5.9856 & 37.5510 & 1.5020 \\
7.0938 & 30.5266 & 1.221 \\
7.2090 & 1.0084
\end{array} \\
& \begin{array}{rrr}
7.9089 & 21.9923 & .8797 \\
9.5709 & 19.9572 & .7983
\end{array}
\end{aligned}
$$

${ }^{-3} \dot{p} p$ elastic at LARGE angle

C ALLABY.....METHERALL (CERN) CERN 68-7 PAGE 580 P.L. 27,149168 C COMHON SCALE ERROR $7 \ldots 12$ GEV OF SEVEN PERCEN OS

2.1 GEV/C, PLAB

T OS/OT ERROR

$2.45802105 .1434 \quad 84.2057$

$\begin{array}{rrr}2.8224 & 166.2016 & 46.648 \\ 3.2079 & 624.3664 & 24.9747\end{array}$

$\begin{array}{lll}3.6135 & 331.3204 & 13.252 \\ 4.0379 & 177.0234 & 7.0809\end{array}$

$\begin{array}{rrr}4.0379 & 177.0234 & 7.0809 \\ 4.4800 & 96.2864 & 3.8515\end{array}$

4.9387
54.6620 2.186

33.7899

$6.0660 \quad 21.3505 \quad .8540$

$6.5704 \quad 17.8720$

$\begin{array}{lll}7.0856 & 15.0709 & \mathbf{9 0 4} \\ 7.7869 & 11.4826 & .6890\end{array}$

$0.5060605 \quad 9.9276650 \quad .5956599$

c'p P ELASTIC
Page 35

C CERN ALLABY ET AL P.L. 25,156

C AT LARGE ANGLE ..... COMPARES Other Data

*.+. DC DATA RESCALED AS PER CERN 68-7 PAGE 588 oxts had INDIV 3 percentisame all pIS each plabi 4. OVerall ISAME all plabI ERRor 7 percent

14.2S GEV/C, PLAB
T OSIDT
$7.6268520 \quad 3.80462170 .1154436$

B. 44256022.94130330 .0953665

$12.5180 \quad 1.6614 \quad 0.0452$

$-3.0000$

C P P ELASTIC

C CERN ALLABY ET AL P.L. 25,156
C AT LARGE ANGLE .... COHPÁRES OTHER DATA

+t++t+ +C DATA RESCALEU AS PER CERN 68-7 PAGE 508

OS/OT IS IN NANOBARNS/GEVEA 2 AND TO BE INCREASED BY $\times 1.07$

$16.9 \mathrm{GEV} / \mathrm{C}$. PLAB

$T$ DS/DT ERROR

$9.1388550 \quad 0.8419603 \quad 0.016755$

$\begin{array}{llll}9.8595021 & 0.6618397 & 0.0251332 \\ 10.3645 & 0.5990 & 0.0209\end{array}$

$11.1175 \quad 0.5069 \quad 0.0168$

$\begin{array}{lll}11.6255 & 0.4315 & 0.0126 \\ 12.3950 & 0.3854 & 0.0168\end{array}$

$\begin{array}{lll}2.9121 & 0.3602 & 0.012\end{array}$

$\begin{array}{lll}13.6924 & 0.3393 & 0.0168 \\ 14.7997 & 0.3142 & 0.0209\end{array}$

$-3.0000$

C P P ELASTIC

C CERN ALLABY EI AL P.L. 25,156

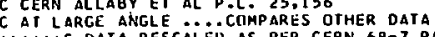

DS 101 IS IN NANOBARNS/GEV**2 AND ID BE INCREASED BY $x 1.00$

$19.3 \mathrm{GEV} / \mathrm{C}$. PLAB

$\begin{array}{cll}\text { t } & \text { OS/OT } & \text { ERROR } \\ 0.6871662 & 0.2484365 & 0.0105\end{array}$

$\begin{array}{ccc}11.0671 & 0.1526 & 0.0095\end{array}$

$\begin{array}{lll}12.7841 & 0.0984 & 0.0073 \\ 17.2483 & 0.0685 & 0.0062\end{array}$

$-3.0000$

C P P FL.ASTIT,

C AT LARGE ANGLE ..... COMPARES OTHER DATA

DAESCALED AS PER CERN S8-7 PAGE 589

DS OT IS IN NANOBARNS/GEV**2
21.3 GEV/C. PLAB

T. DS/OT

11: $3448 \quad 0.0723 \quad 0.0059$

$\begin{array}{lll}12.5824 & 0.0444 & 0.0035 \\ 14.1734 & 0.0332 & 0.0026\end{array}$

$\begin{array}{lll}14.1734 & 0.0332 & 0.0026 \\ 18.1219 & 0.0200 & 0.0030\end{array}$

P.3.0000

C OREAR ET AL P.R. 138,1651651 LARGE ANGLE LOTS OF PLAB $C 20$ PERCENT SYSTEMAT TC ERR
OSIDT IS IN NANORARNS/GEV**2

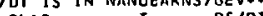

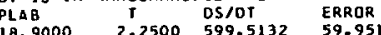

$\begin{array}{rrrr}18.9000 & 2.2500 & 599.5132 & 59.9513 \\ 13,0000 & 2.2800 & 1694.2577 & 1 \times 9.4252 \\ 11.0000 & 3.1500 & 1007.7024 & 100.7702\end{array}$

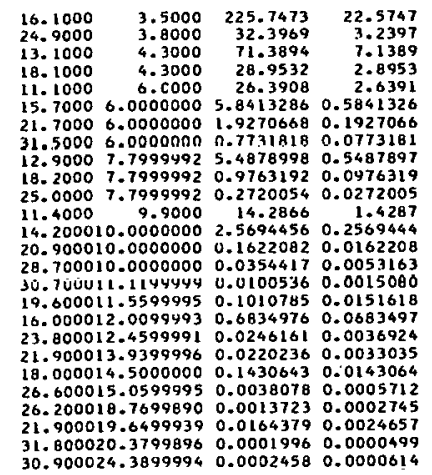

S\$ ISSNEUTRON ANTIPROTON SCATIERING, TOTAL CROSS SECTION ONLY C PHAR NEUTRON ELASTIC SCATTERING

C IHEP-CERN COLLABORATION, OC TCBER, 1969
C NOW PUBLISHEU IN PHYS. LETTERS 308 . .

$46.00 \quad 1.700 \quad$ MB.

20. GEV/C. PLAB

45. $30 \quad 1.100$

25.

30. 1.100

$45.90 \quad 1.100$

*** 1.100

$\begin{array}{ll}43.2 & 1.100 \\ 40 . & 1.100\end{array}$

$45.0 \quad 1.100$

$44.1 \quad 1.200$

-4 .

SSSSSNEUTRON PROTON ELASTIC SCATTERING

C N P ELASTIC.

C 2.0 TO $7.0 \mathrm{GEV} / \mathrm{C}$... 
This report was prepared as an account of Government sponsored work. Neither the United States, nor the Commission, nor any person acting on behalf of the Commission:

A. Makes any warranty or representation, expressed or implied, with respect to the accuracy, completeness, or usefulness of the information contained in this report, or that the use of any information, apparatus, method, or process disclosed in this report may not infringe privately owned rights; or

B. Assumes any liabilities with respect to the use of, or for damages resulting from the use of any information, apparatus, method, or process disclosed in this report.

As used in the above, "person acting on behalf of the Commission" includes any employee or contractor of the Commission, or employee of such contractor, to the extent that such employee or contractor of the Commission, or employee of such contractor prepares, disseminates, or provides access to, any information pursuant to his employment or contract with the Commission, or his employment with such contractor. 